\title{
Andreas Wagner
}

\section{Fördert die Exzellenzinitiative soziale Ungleichheit bei der Hochschulwahl?}

\section{Untersuchung sozialer Folgen und Determinanten eines staatlichen \\ Förderprogramms}

\author{
International Centre for \\ Higher Education Research Kassel \\ Universität Kassel
}

Kassel 2015

INCHER Working Paper 5

U N I K A S S E L

V $\begin{array}{lllllll} & R & S & \text { I } & T & \text { "A" } & T\end{array}$ 


\section{Andreas Wagner}

\section{Fördert die Exzellenzinitiative soziale Ungleichheit bei der Hochschulwahl?}

Untersuchung sozialer Folgen und Determinanten eines staatlichen Förderprogramms

International Centre for

Higher Education Research Kassel

Universität Kassel

Kassel 2015

INCHER Working Paper 5 


\section{Inhalt}

Zusammenfassung 2

1 Einleitung 3

2 Die Exzellenzinitiative 4

3 Theoretischer Hintergrund 4

3.1 Die Hochschulwahl als rationale Bildungsentscheidung 4

Erklärung der Wahl einer Zukunftskonzept-Universität vornehmlich
durch statushohe Akteure

$4 \quad$ Forschungsstand $\quad 6$

4.1 Internationaler Forschungsstand $\quad 6$

$\begin{array}{lll}4.2 & \text { Nationaler Forschungsstand } & 7\end{array}$

5 Methodische Herangehensweise $\quad 8$

$\begin{array}{lll}5.1 & \text { Datengrundlage } & 8\end{array}$

5.2 Untersuchungsdesign 9

$\begin{array}{lll}5.3 & \text { Stichprobenabgrenzung und fehlende Werte } & 10\end{array}$

$\begin{array}{lll}5.4 & \text { Operationalisierung zentraler Variablen } & 10\end{array}$

$\begin{array}{lll}\text { 5.4.1 Exzellenzstatus } & 10\end{array}$

5.4.2 Soziale Herkunft 11

$\begin{array}{lll}\text { 5.4.3 Elterliche Bildung } & 13\end{array}$

6 Hypothesentests 14

6.1 Querschnittdesign: Determinanten der Wahl einer Exzellenzuniversität 14

$\begin{array}{lll}\text { 6.1.1 Deskriptive Ergebnisse } & 14\end{array}$

6.1.2 Multivariate Analysen 16

6.2 Längsschnittdesign: Die Wirkung der Exzellenzinitiative auf die
Hochschulwahl von Studierenden mit hohem SÖS

$\begin{array}{lll}7 & \text { Zwischenfazit } & 20\end{array}$

$8 \quad$ Test zeitstabiler SÖS-Selektionseffekte auf Zukunftskonzept-Universitäten 21

$9 \quad$ Test einer Prestigewirkung der SÖS-selektiven Zukunftskonzept-Universitäten 23

10 Einschätzung der Ergebnisse $\quad 25$

$\begin{array}{ll}\text { Literaturverzeichnis } & 27\end{array}$ 


\section{Zusammenfassung}

Dieser Beitrag nutzt hauptsächlich die Daten des Deutschen Studierendensurveys der AG Hochschulforschung der Universität Konstanz, um in einem quasi-experimentellen Untersuchungsdesign einem eventuellen Kausaleffekt der Exzellenzinitiative auf die Hochschulwahl von Studierenden mit hohem sozioökonomischen Status (SÖS) nachzugehen: Zwar bevorzugen Studierende mit hohem SÖS signifikant wahrscheinlicher Universitäten der prestigereichen Förderlinie 3 (Zukunftskonzept) als Studierende mit niedrigem SÖS, ein FixedEffects-Regressionsmodell misst allerdings keinen signifikanten Effekt einer Förderung durch die Exzellenzinitiative auf die sozioökonomische Zusammensetzung der Studierendenschaft einer Universität über die Zeit. Daten, die vor Veröffentlichung der ersten Förderentscheidungen im Oktober 2006 erhoben wurden, offenbaren darüber hinaus einen signifikanten zeitstabilen SÖS-Selektionseffekt auf Universitäten, die in den Folgejahren eine Auszeichnung durch die Förderlinie 3 erhalten haben. Des Weiteren wird in diesen Daten eine Korrelation zwischen der Prestigewirkung einer Universität und ihrer SÖS-Selektivität gefunden. Die Ergebnisse weisen darauf hin, dass die Exzellenzinitiative keine SÖS-Selektionseffekte verursacht, sondern bereits vorhandene zeitstabile Prestigedifferenzen im Hochschulsystem abbildet, welche zudem mit sozialer Ungleichheit in den Studierendenkompositionen einhergehen. 


\section{$1 \quad$ Einleitung}

Im Zuge der Bildungsexpansion ist die Zahl der Hochschulabsolventen im tertiären Bildungssystem gestiegen und es haben sich neue Wege zum Erwerb der Hochschulreife eröffnet (Müller et al. 2011: 293, 296). Gleichzeitig hat sich die Chance auf eine Hochschulbildung für bildungsferne Gruppen erhöht: So ist der Anteil Studienberechtigter, deren Eltern maximal einen Hauptschulabschluss besitzen, zwischen den Absolventenkohorten 1976 und 2008 von etwa $17 \%$ auf über $30 \%$ gestiegen (Schindler 2014: 156). Gleichzeitig ist unter dem Eindruck einer Inflation von Hochschulbildungszertifikaten ein Trend zu vertikaler Differenzierung zwischen Hochschulen nach Qualität und Prestige durch systemexterne Evaluationsmaßnahmen zu erkennen (z.B. CHE-Ranking, Exzellenzinitiative). Eine Folge dieser vertikalen Differenzierung könnten sozial ungleiche Bildungserträge der Hochschulabsolventen auf dem Arbeitsmarkt sein, wie es etwa in anglo-amerikanischen Hochschulsystemen traditionell der Fall ist (Bowen und Bok 1998; Carnevale und Rose 2003). Eine soziologische Theorie rationaler Bildungsentscheidungen (Erikson und Jonsson 1996, Breen und Goldthorpe 1997) bildet in diesem Beitrag die Grundlage für die Hypothese eines Selektionsprozesses, nach dem Studierende mit hohem sozioökonomischen Status (SÖS) in Erwartung einer höheren Bildungsrendite bevorzugt Universitäten wählen, die durch die Exzellenzinitiative ausgezeichnet wurden.

Trotz intensiver Diskussion möglicher sozialer Folgen der Exzellenzinitiative (vgl. z.B. Hartmann 2012) ist derzeit nur wenig empirische Evidenz kausaler Wirkmechanismen dieser umstrittenen Maßnahme vorhanden. Selektionseffekte nach sozialer Herkunft waren insbesondere in Bezug auf den Zugang zum Hochschulsystem Untersuchungsgegenstand der nationalen Bildungsforschung (e.g. Arbeitsgruppe Hochschulforschung der Universität Konstanz 2007, Becker und Hecken 2007, Müller et al. 2011; Schindler und Reimer 2010; Schindler und Lörz 2012), weniger hinsichtlich des Zugangs zu Hochschulen mit speziellen Merkmalen. Doch gerade weil das deutsche Hochschulsystem durch eine traditionelle Standardisierung der Bildungsqualität und damit durch eine scheinbar sicher gegebene Gleichwertigkeit aller Hochschulzertifikate auf dem Arbeitsmarkt charakterisiert werden kann (Allmendinger 1989: 236 ff.), besitzt hierzulande die Frage nach sozialer Ungleichheit in der Universitätswahl als eventueller Folge von Qualitäts- bzw. Prestigedifferenzen besondere Forschungsrelevanz. Darüber hinaus hat die Fragestellung internationale Bedeutung: Sollte ein Kausaleffekt identifiziert werden, so scheint eine Übertragung sozialer Folgen politisch induzierter Differenzierungsmaßnahmen auf andere Staaten mit traditionell standardisierten Hochschulsystemen keinesfalls abwegig. Nationen, die im globalen Wettbewerb um Humankapital attraktiv für ausländische Studierende und Wissenschaftlerinnen und Wissenschaftler erscheinen möchten, stehen unter Druck, Spitzenuniversitäten vorzeigen zu können, die den 
Vergleich in den Output-Indikatoren gängiger Evaluationsinstrumente bestehen (Hazelkorn 2007; Marginson 2009).

\section{Die Exzellenzinitiative}

Die Exzellenzinitiative wurde im Jahr 2005 mit einer Vereinbarung zwischen Bund und Ländern beschlossen (Bundesministerium für Bildung und Forschung 2005). Das Förderprogramm ist auf Universitäten begrenzt, d.h. Fachhochschulen sind ausgenommen (ebd.). Ziel des Wettbewerbsverfahrens ist, Universitäten mit hohem Leistungspotenzial zu identifizieren und deren „wissenschaftliche Aktivitäten“ daraufhin gezielt finanziell zu fördern (ebd.: §1). Als Legitimationsgrundlage der Bevorteilung einzelner Universitäten dient im gegenwärtigen Diskurs die Sicherung globaler Wettbewerbsfähigkeit über eine hochschulspezifische Konzentration von Humankapital und strukturellen Ressourcen (Bundesministerium für Bildung und Forschung 2005; 2012). Insgesamt standen im Zeitraum 2006 bis 2011 Mittel in Höhe von 1,9 Mrd. € zur Verfügung (Bundesministerium für Bildung und Forschung 2005: §2). Die Ausschreibung der ersten Programmphase startete im August 2005 und im Oktober der Jahre 2006 und 2007 wurden die Förderentscheidungen veröffentlicht (ebd.).

Die Förderung durch die Exzellenzinitiative ist in drei Linien unterteilt: 1. „Graduiertenschule“ (Förderung von Doktoranden), 2. „Exzellenzcluster“ (zusätzliche themenspezifische Forschungsförderung) und 3. „Zukunftskonzept“ (Auszeichnung eines strategischen Forschungskonzepts der Universität für die Zukunft) (ebd.). Die Förderlinie 3 „Zukunftskonzept“ stellt die finanzintensivste (ebd.), begehrteste und prestigeträchtigste Förderlinie dar, was sich zum einen in der spezifischen Rhetorik des offiziellen Titels „Exzellenzuniversität“ und zum anderen in der zum anglo-amerikanischen Hochschulsystem äquivalent verwendeten Bezeichnung „Eliteuniversität“ (vgl. z.B. Die Welt 2012) äußert. Im Einklang damit steht, dass die Förderung über Linie 3 „Zukunftskonzept“ am voraussetzungsreichsten ist: Sie kann nur dann erfolgen, wenn bereits mindestens ein „Exzellenzcluster" und eine „Graduiertenschule“ eingeworben wurde (Bundesministerium für Bildung und Forschung 2005).

\section{Theoretischer Hintergrund}

\subsection{Die Hochschulwahl als rationale Bildungsentscheidung}

Es ist nicht unumstritten, ob die Hochschulwahl als rationale Entscheidung modelliert werden kann. So zeigen Befunde, dass die Hochschulwahl zwar unter Betrachtung finanzieller Kos- 
ten geschieht, jedoch einen langfristigen und komplexen Prozess darstellt, in dem in jeder Phase verschiedenste Faktoren die Entscheidung beeinflussen (Sojkin et al. 2012). An anderer Stelle wird aufgezeigt, dass weniger als die Hälfte der angehenden Studierenden als aktiv Information suchend eingestuft werden können, selbst wenn die Wahrscheinlichkeit für eine Informationssuche mit steigendem SÖS zunimmt (Menon et al. 2007). Des Weiteren sind nicht-traditionelle Studierende in Großbritannien, die eine prestigereiche Universität gewählt haben, weniger rational kalkulierend, sondern lassen sich vielmehr von emotionalen Eindrücken leiten, welche eher auf eine unreflektierte Entscheidung, gleichermaßen geprägt durch „Abschreckung und Faszination“ [deutsche Übersetzung], schließen lassen (Baker und Brown 2007: 377, 385 ff.).

Auf der anderen Seite werden aktiv Information suchende Akteure und deren Kosten/Nutzenabwägungen in der Hochschulwahlentscheidung stets vorausgesetzt, wenn Hochschulrankings als Informationssystem unter Marktbedingungen, also als ein Werben um „Kunden“, aufgefasst werden (e.g. Sarrico et al. 1997; Eff et al. 2012). Akteure suchen insbesondere dann aktiv Information und wägen Kosten und Nutzen einer Universität ab, wenn sie die Universität als primäres Wahlkriterium ansehen und nicht einen spezifischen Studiengang, für den sie sich interessieren (Tavares und Cardoso 2013). Besonders vor dem Hintergrund, dass der Ruf der Hochschule als Hochschulwahlmotiv in Deutschland an Bedeutung zunimmt (Lörz und Quast 2011: 4) ist dieser Befund als relevant einzuschätzen. Außerdem spricht für die Anwendung der Theorie Rationaler Wahl (RCT) in Form der SEU-Theorie (Erikson und Jonsson 1996, Breen und Goldthorpe 1997, s.a. für einen Überblick: Diekmann und Voss 2004, Diefenbach 2009), dass in Deutschland die erwarteten Kosten eines Wohnortwechsels und die bisher erreichten Schulnoten (Erfolgserwartungen) die Wahl hoch gerankter, prestigereicher Hochschulen determinieren (ebd.).

\subsection{Erklärung der Wahl einer Zukunftskonzept-Universität vornehmlich durch statushohe Akteure}

Der angenommene Hochschulwahlmechanismus kann allgemein als prestigegeleitet charakterisiert werden: Setzt man demgegenüber Akteure voraus, die unter begrenzter subjektiver statt perfekter objektiver Rationalität Kosten-/ Nutzenwahrnehmungen vornehmen (s.a. Esser 1999), bleibt dem Individuum die tatsächliche Lehrqualität einer Bildungsalternative als Entscheidungsgrundlage verwehrt: Gemäß der SEU-Theorie (Subjectively Expected Utility) (Erikson und Jonsson 1996, Breen und Goldthorpe 1997) bestimmen die akteurspezifische subjektive Erfolgs- (p), Nutzen- (u) und Kostenerwartung (c) einer Bildungsoption, in diesem Fall des Studiums an einer Zukunftskonzept- bzw. „Exzellenzuniversität“, über die Funktion 
p*u-c die Wahlwahrscheinlichkeit des Bildungsgangs (s.a. Erikson und Jonsson 1996). Die Ausprägungen der Entscheidungsparameter variieren dabei je nach sozioökonomischem Status des Akteurs und lassen letztlich die Wahlwahrscheinlichkeit der Akteure höherer sozialer Klassen steigen (ebd.): So haben Akteure mit hohem SÖS aufgrund ihrer besseren Schulleistungen in der Vergangenheit (Esping-Andersen 2004) die Überzeugung gewonnen, den Abschluss an einer Zukunftskonzept- bzw. Exzellenzuniversität schaffen zu können (s.a. Erikson und Jonsson 1996), welchem die Akteure als Folge der Selektivität und Qualität suggerierenden Exzellenzrhetorik hohe Leistungsanforderungen unterstellen. Weiterhin ist der subjektiv erwartete Bildungsnutzen (u) eines Studiums an einer ZukunftskonzeptUniversität, welcher in der Vermeidung intergenerationalen Statusverlusts besteht (s.a. ebd.; Breen und Goldthorpe 1997), für Akteure mit hohem SÖS besonders hoch. Dem liegt die subjektive Annahme einer gegenüber Akteuren mit niedrigem SÖS erhöhten Chance auf eine einkommensstarke und prestigereiche Berufsposition nach Abschluss des eigenen Studiums zugrunde (vgl. Erikson und Jonsson 1996; Breen und Goldthorpe 1997). Diese Erwartung der Akteure ergibt sich wiederum aus der Antizipation einer besseren Bewertung des Hochschulbildungszertifikats durch den späteren Arbeitgeber, welcher den Hochschulstandort als Indikator für die Kompetenzen des Hochschulabsolventen heranzieht. ${ }^{1}$ Akteure mit hohem SÖS schätzen zudem ihre Mobilitätskosten (c) geringer ein (ebd.): Finanzielle Mittel etwa, die die Eltern für einen Umzug des Akteurs an den Ort einer Exzellenzuniversität aufwenden müssten, wiegen für diese Gruppe weniger schwer.

Aus den vorangehenden Ausführungen kann folgende Forschungshypothese abgeleitet werden: Es besteht ein Kausaleffekt der Exzellenzinitiative auf die Wahl einer Zukunftskonzept-Universität durch Akteure mit hohem SÖS.

\section{$4 \quad$ Forschungsstand}

\subsection{Internationaler Forschungsstand}

Im anglo-amerikanischen Raum kommt der Erforschung sozialer Ungleichheit im Zusammenhang mit vertikaler Differenzierung zwischen Hochschulen aufgrund der strukturellen Unterschiede der Hochschulsysteme traditionell eine bedeutende Rolle zu. Hinsichtlich der Wahrnehmung von Hochschulevaluationsverfahren wird für die USA zunächst ein Effekt der Position im „U.S. News \& World Report's Best Colleges College-Ranking“ auf die tatsächli-

\footnotetext{
1 Aus Sicht der in der Ökonomie verwurzelten Signaltheorie (Spence 1973) sieht der Arbeitgeber den Hochschulstandort auf dem Bildungszertifikat als Signal für eine hohe Produktivität der Absolventin oder des Absolventen auf dem Arbeitsmarkt an.
} 
che Reputation einer Hochschule gefunden (e.g. Bastedo und Bowman 2010). Weiter unterscheidet sich die soziale Zusammensetzung der Studierenden nach dem Prestige der Hochschule: So wird ein Effekt der Position im U.S. News \& World Reports Best Colleges auf die sozioökonomische Zusammensetzung der Studierendenschaft und ihre ethnische Komposition (Meredith 2004) sowie den durchschnittlichen SAT (Scholastic Achievement Test)-Score (Monks und Ehrenberg 1999) gefunden. Der eingeschränkte Zugang bildungsferner Gruppen zu prestigereichen Hochschulen lässt sich zu einem Teil über deren vorherigen geringen Schulerfolg (Valencia und Aburto 1991; Fleming 1990) erklären. Damit steht im Einklang, dass das Ausmaß, in dem angehende Studierende College-Rankings und damit der Reputation einer Hochschule nach Eigenaussagen hinsichtlich der Hochschulwahl Beachtung schenken, mit deren Schulerfolg korreliert ist (McDonough et al. 1998). Doch auch wenn kognitive Kompetenzen der Studierenden eine einflussreiche Determinante für die Selektion der Studierenden auf Colleges von hoher Qualität bzw. hoher Rankingposition sind, kann der Effekt der sozialen Herkunft nur zu einem Teil über diese Faktoren erklärt werden (z.B. Karabel und Astin 1975). Unter statistischer Kontrolle der kombinierten mathematik- und sprachbezogenen SAT-Scores besteht zudem ein Selektionseffekt des Familieneinkommens auf den Besuch einer privaten Universität aus der COFHE (Consortium on Financing Higher Education)-Gruppe (McPherson et al. 1991). Soziale Ungleichheit in den Studierendenkompositionen unterschiedlich selektiver Colleges und Universitäten hat sich im USamerikanischen Bildungssystem über die letzten Jahrzehnte sogar noch ausgeweitet (Astin und Osuerga 2004). Gleichzeitig zeichnen sich hoch gerankte, selektive Hochschulen neben einer besseren Lehre durch bessere Arbeitsmarktchancen ihrer Absolventen aus (Bowen und Bok 1998; Carnevale und Rose 2003). Dabei wirkt sich der Besuch privater EliteColleges (Brewer et al. 1999) und die Leistungsselektivität eines Colleges (Datcher-Loury und Garman 1995) auf das spätere Arbeitsmarkteinkommen der Studierenden positiv aus. Beispiele aus anderen Ländern bestätigen diesen Zusammenhang: Der Besuch reputationsstarker chinesischer Universitäten, die besondere finanzielle staatliche Förderung erhalten, hat einen positiven Effekt auf das spätere Arbeitsmarkteinkommen der Absolventen im Land (Hartog et al. 2010), während in Großbritannien die Absolventen prestigereicher EliteUniversitäten überwiegend die berufliche Elite stellen (Williams und Filippakou 2010).

\subsection{Nationaler Forschungsstand}

Was Deutschland betrifft, zeigt sich, dass, sobald das Abitur erreicht wurde, die Entscheidung zwischen Fachhochschule und Universität von der sozialen Herkunft abhängt (Müller et al. 2011). Studierende, die eine Universität besuchen, müssen also innerhalb des deutschen 
Hochschulsystems bereits als eine selektive Gruppe angesehen werden. Prestige ist als Hochschulwahlmotiv dabei nicht zu unterschätzen: 2009/10 sagten $61 \%$ der Befragten aus, dass innen ein guter Ruf der Hochschule wichtig für die Hochschulwahl sei (Willich et al. 2011). Es wird außerdem dargelegt, dass in Deutschland der Ruf der Hochschule über die letzten drei Erhebungswellen für alle Gruppen sozialer Herkunft ein immer wichtigeres Hochschulwahlmotiv geworden ist, während sich bestehende leichte Differenzen zwischen den SÖS-Gruppen diesbezüglich allerdings nicht vergrößert haben (Lörz und Quast 2011). Soziale Selektionseffekte nach Bildungsherkunft werden auf Hochschulen gefunden, die laut CHE-Ranking einen guten Ruf unter Professorinnen und Professoren genießen (Lörz und Quast 2011). Neben der Position im Shanghai-Ranking (Helbig und Ulbricht 2010) wird auch die Förderung durch die Exzellenzinitiative als ein Faktor identifiziert, der besonders die Hochschulwahl leistungsstarker Studierender beeinflusst (Helbig und Ulbricht 2010; Horstschräer 2011). Der Effekt der Exzellenzinitiative hält dabei statistischer Kontrolle zeitstabiler Prestigedeterminanten, also unbeobachteter Heterogenität zwischen Hochschulen über die Zeit, stand (Horstschräer 2011). Darüber hinaus wird aufgezeigt, dass eine Veränderung im Exzellenzstatus über die Zeit einen Anstieg der Bewerberzahlen um 19\% zur Folge hat (ebd.), was für die öffentliche Wahrnehmung des Exzellenzstatus und eine entsprechende Wertzuschreibung durch die Studierenden spricht.

Eine weitere Determinante der Hochschulwahl ist nach wie vor die Entfernung zur Hochschule (Büttner et al. 2003; Denzler und Wolter 2010). Die Distanz zur Universität kann in diesem Zusammenhang als eine RCT-Kostendeterminante aufgefasst werden, welche Gruppen niedriger sozialer Herkunft davon abhält, eine weit entfernte Hochschule zu wählen (Denzler und Wolter 2010; Lörz und Quast 2011). Unterschiede in der Wahl einer prestigereichen Hochschule nach sozialer Herkunft lassen sich neben geringeren Schulleistungen auf eingeschränkte Mobilitätsmöglichkeiten bzw. hohe Mobilitätskosten dieser Gruppe zurückführen (Lörz und Quast 2011).

\section{$5 \quad$ Methodische Herangehensweise}

\subsection{Datengrundlage}

Der Deutsche Studierendensurvey der Universität Konstanz, dessen Erhebungen in Abständen von zwei bis drei Jahren durchgeführt werden, stellt die primäre Datengrundlage für die folgenden Analysen dar. Es handelt sich um Trenddaten auf Individualebene, die Studierende unterschiedlicher Semester auf verschiedenen staatlichen Hochschulen erfassen. Im Fokus dieses Beitrages steht ein eventueller Kausaleffekt der Veröffentlichung der ersten bei- 
den Förderentscheidungen im Oktober 2006 und Oktober 2007 (Phase 1 der Exzellenzinitiative) auf die Hochschulwahl von Studierenden mit hohem SÖS. Genutzt werden dazu die vier Messwellen der Wintersemester 2000/01, 2003/04, 2006/07 und 2009/10, wobei die zwei Veröffentlichungstermine als experimenteller Stimulus zeitlich zwischen die beiden letzten Wellen fallen. Für 16 der im Datensatz verfügbaren Universitäten stehen Daten aller vier Messwellen zur Verfügung. Sieben davon werden ab 2006 gefördert, zwei von diesen sieben bekommen 2007 nochmals einen höheren Exzellenzstatus zugewiesen. Zwei Universitäten werden erst ab 2007 gefördert. Weitere sieben Universitäten erhalten von der Exzellenzinitiative keine Fördergelder und dienen als Kontrollgruppe.

\subsection{Untersuchungsdesign}

Der Test der Forschungshypothese erfolgt in zwei Schritten: Zunächst wird in einem querschnittlichen Untersuchungsdesign über eine multinomiale logistische Regression geprüft, ob Studierende mit vorteilhafter sozialer Herkunft wahrscheinlicher durch die Exzellenzinitiative geförderte Universitäten gegenüber nicht geförderten bevorzugen als Studierende mit niedriger sozialer Herkunft. Im zweiten Schritt werden alle vier Wellen in die Schätzung einbezogen: Dieses längsschnittliche Design kann als natürliches Experiment beschrieben werden, wobei wie bereits angedeutet die Wellen 2000/01, 2003/04, 2006/07 den Pretest und Welle 2009/10 den Posttest darstellt. Eine OLS (Ordinary Least Squares)-Regression mit FixedEffects (FE) (vgl. z.B. Halaby 2004) in Form von Kontrollvariablen für die einzelnen Universitäten schätzen Effekte einer Förderung durch die Exzellenzinitiative für jede Förderlinie auf die Studierendenkomposition einer Universität über die Zeit. Durch statistische Kontrolle der universitären Fixed-Effects wird dabei die zur Effektschätzung genutzte Varianz auf die Universitätsebene beschränkt (Within-Schätzer) (vgl. z.B. Brüderl 2010). Studierende werden zu diesem Zweck lediglich innerhalb einer Universität über die Zeit verglichen, nicht zwischen Universitäten. Auf diese Weise wird versucht, eine nicht zufällig erfolgende Zuordnung der Untersuchungseinheiten in die Versuchsgruppen, in diesem Fall der Universitäten, in die Förderlinien der Exzellenzinitiative zu kompensieren. Unbeobachtete und zeitstabile Universitätsmerkmale, wie etwa Bundesland oder zeitkonstantes Prestige der Organisation, die sich gegebenenfalls gleichermaßen auf die Zuweisung eines Exzellenzstatus und die Studierendenkomposition einer Universität auswirken, verzerren bei dieser Vorgehensweise nicht die Parameterschätzung. 


\subsection{Stichprobenabgrenzung und fehlende Werte}

Die vier genutzten Wellen beinhalten 34045 Studierende. Weil sich die Exzellenzinitiative auf Universitäten beschränkt, werden zunächst alle Studierenden, die eine Fachhochschule besuchen, ausgeschlossen, was die Fallzahl auf 27703 reduziert. Weiterhin müssen Studierende im Alter von unter 20 Jahren und über 30 Jahren aus der Stichprobe entfernt werden, sodass diese letztlich noch 24433 Studierende beinhaltet. Der Grund hierfür liegt in der Operationalisierung der Variable sozioökonomischer Status (siehe unten). Für die Rekonstruktion der individuellen Hochschulwahlzeitpunkte im ersten Schritt (Querschnittdesign) wird die im WS 2009/10 berichtete Anzahl an studierten Fachsemestern herangezogen. Weil im Fall von Studienunterbrechungen, Praktika, Studienfachwechseln und Hochschulwechseln nicht ausgeschlossen werden kann, dass das Studium bereits vor Bekanntgabe der Förderentscheidungen begonnen wurde, ergeben sich weitere 1717 fehlende Werte auf der Variable „Exzellenzstatus der Universität bei Studienbeginn“. Bei keiner Regressionsanalyse übersteigt der Anteil der durch fehlende Werte in den unabhängigen Variablen verlorenen Fälle $13 \%$ der Stichprobe. Aufgrund dieses als akzeptabel eingeschätzten Verhältnisses wurden die Analysen lediglich mit den Fällen durchgeführt, die keine fehlenden Werte in den unabhängigen Variablen aufweisen (Listwise Deletion). Nichtsdestotrotz wurde in Betracht gezogen, dass ein vorliegender MAR (Missing At Random)- bzw. MNAR (Missing Not At Random)-Mechanismus die Schätzungen unabhängig vom Anteil der verlorenen Fälle verzerren kann. Mithilfe von T-Signifikanztests konnte zumindest eine besonders hohe Zahl signifikanter Korrelationen zwischen beobachteten Merkmalen und dem Auftreten fehlender Werte ausgeschlossen werden.

\section{$5.4 \quad$ Operationalisierung zentraler Variablen}

\subsubsection{Exzellenzstatus}

Der Exzellenzstatus einer Universität berücksichtigt durch eine kategoriale Operationalisierung alle drei Förderlinien der Exzellenzinitiative und ist über die höchste Förderlinie definiert, über die eine Universität zu einem Zeitpunkt gefördert wird. Die durchschnittlich in Phase 1 bewilligten finanziellen Mittel einer Fördermaßnahme korrelieren positiv mit der Nummer der Förderlinie: FL 1: 3,4 Mio. €, FL 2: 19,1 Mio. €, FL 3: 37,7 Mio. €. (eigene Berechnung auf der Grundlage Leibfried 2010: 15). Gleichzeitig nimmt mit der Nummer der Förderlinie die Anzahl bewilligter Maßnahmen nach Förderlinie ab (FL 1: 39; FL 2: 37; FL 3: 9) (ebd.). Diese Zusammenhänge lassen eine Hierarchie des Exzellenzstatus hinsichtlich 
seiner Attraktivität für Studierende mit hohem SÖS entlang der Dimensionen Exklusivität und Förderumfang vermuten. Die Berücksichtigung dessen in Form einer kategorialen Operationalisierung des Exzellenzstatus ermöglicht einen Test dieser Annahme.

\subsubsection{Soziale Herkunft}

Der familiäre SÖS der Studierenden wird über den ISEI den ISEI (International SocioEconomic Index of Occupational Status) (Ganzeboom et al. 1992) desjenigen Elternteils definiert, das in diesem die höchste Ausprägung besitzt (Dominance-Model). ISEI ist metrisch skaliert und beruht auf ISCO (International Standard Classification of Occupations) (hier ISCO 1988), die international unterschiedlichen Berufsgruppen metrische Prestigewerte zuweist (ebd.: $15 \mathrm{ff}$.). Darüber hinaus fließt in ISEI das Einkommen der Eltern und deren Berufsausbildung ein (ebd.: 17 ff.). Die Operationalisierung der sozialen Herkunft als SÖS durch ein valides Maß ist vor dem Hintergrund der theoretischen Implikation des intergenerationalen Statuserhalts ein zentrales Ziel dieses Forschungsbeitrags.

Der Datensatz des Deutschen Studierendensurveys der Universität Konstanz enthält selbst keine ISEI-Werte, was den Hypothesentest erschwert. Entsprechende Werte der EItern von Studierenden aus dem Datensatz der Allgemeinen Bevölkerungsumfrage der Sozialwissenschaften (ALLBUS) wurden differenziert nach deren feingegliederter Stellung im Beruf (STIB) und dem Alter der Studierenden berechnet und dem Datensatz zugespielt. Personen, die jünger als 20 Jahre alt sind, wurden aus der Stichprobe gelöscht, weil ALLBUS den Nichterwerbstätigkeitsstatus von Schülern und Studenten als eine Kategorie erfasst und die Wahrscheinlichkeit relativ gering eingeschätzt wird, dass sich noch Schüler in der Stichprobe befinden. Die obere Altersgrenze wurde unter Berücksichtigung der Altersverteilungen beider Datensätze auf 30 Jahre gesetzt. Tabelle 1 stellt die arithmetischen ISEI-Mittelwerte, deren Berechnung auf Grundlage einer gepoolten Stichprobe der ALLBUS-Wellen 2000, 2002, 2004, 2006, 2008 und 2010 vorgenommen wurde, und deren zugrundeliegende Fallzahlen dar. Weil ISEI in ALLBUS ab der Welle 2000 verfügbar ist, gehen die vier Wellen des Deutschen Studierendensurveys mit zeitlicher Entsprechung in die Analysen ein. 
Tabelle 1 Berechnung der ISEI-Mittelwerte und Zuspielung aus dem ALLBUSDatensatz

\begin{tabular}{|c|c|c|c|c|c|c|c|c|c|c|}
\hline & \multicolumn{9}{|c|}{ Alter der Studierenden (Befragungspersonen) } \\
\hline & & $\begin{array}{l}20-21 \\
\text { Jahre }\end{array}$ & $\begin{array}{l}22-23 \\
\text { Jahre }\end{array}$ & $\begin{array}{l}24-26 \\
\text { Jahre }\end{array}$ & $\begin{array}{l}27-30 \\
\text { Jahre }\end{array}$ & & $\begin{array}{l}20-21 \\
\text { Jahre }\end{array}$ & $\begin{array}{l}22-23 \\
\text { Jahre }\end{array}$ & $\begin{array}{l}24-26 \\
\text { Jahre }\end{array}$ & $\begin{array}{l}27-30 \\
\text { Jahre }\end{array}$ \\
\hline \multirow{3}{*}{$\begin{array}{c}01 \text { ungelernte/ } \\
\text { angelernte } \\
\text { Arbeiter }\end{array}$} & Vater & 27,57 & 28,00 & 25,91 & 25,00 & Mutter & 29,00 & - & 20,00 & 31,00 \\
\hline & N1 & 7 & 5 & 11 & 2 & & 8 & 0 & 2 & 1 \\
\hline & N2 & 140 & 153 & 204 & 113 & & 147 & $226 X$ & 234 & 141 \\
\hline \multirow{3}{*}{$\begin{array}{l}02 \text { Facharbei- } \\
\text { ter-/unselbst- } \\
\text { ständige } \\
\text { Handwerker }\end{array}$} & Vater & 35,81 & 34,10 & 31,81 & 32,36 & Mutter & 30,20 & 41,00 & 40,00 & 30,5 \\
\hline & N1 & 32 & 31 & 16 & 14 & & 5 & 3 & 1 & 2 \\
\hline & N2 & 621 & 691 & 762 & 419 & & 183 & 233 & 255 & 160 \\
\hline \multirow{3}{*}{$\begin{array}{c}03 \\
\text { Meister } \\
\text { /Poliere }\end{array}$} & Vater & - & 36,83 & 31,67 & 37,00 & Mutter & - & - & - & - \\
\hline & N1 & 0 & 6 & 3 & 1 & & 0 & 0 & 0 & 0 \\
\hline & N2 & $141 X$ & 179 & 166 & 112 & & $15 X$ & $20 X$ & $19 X$ & $16 X$ \\
\hline \multirow{3}{*}{$\begin{array}{c}04 \\
\text { ausführende } \\
\text { Angestellte }\end{array}$} & Vater & 41,67 & 34,86 & 34,40 & - & Mutter & 49,57 & 44,07 & 47,14 & 43,00 \\
\hline & N1 & 3 & 7 & 5 & 0 & & 6 & 14 & 7 & 3 \\
\hline & N2 & 202 & 208 & 213 & $83 X$ & & 645 & 732 & 845 & 445 \\
\hline \multirow{3}{*}{$\begin{array}{c}05 \text { qualifizierte } \\
\text { Angestellte }\end{array}$} & Vater & 58,38 & 57,55 & 55,54 & 60,87 & Mutter & 50,89 & 53,06 & 50,88 & 53,89 \\
\hline & N1 & 45 & 40 & 43 & 23 & & 45 & 52 & 43 & 18 \\
\hline & N2 & 857 & 954 & 961 & 449 & & 2132 & 2282 & 2477 & 1098 \\
\hline \multirow{3}{*}{$\begin{array}{c}06 \\
\text { leitende } \\
\text { /hochqual. } \\
\text { Angestellte }\end{array}$} & Vater & 63,33 & 62,60 & 61,33 & 68,00 & Mutter & 70,00 & - & 44,50 & - \\
\hline & N1 & 12 & 5 & 6 & 1 & & 1 & 0 & 2 & 0 \\
\hline & N2 & 1554 & 1675 & 1838 & 802 & & 612 & $710 \times$ & 720 & 284 \\
\hline \multirow{3}{*}{$\begin{array}{c}07 \text { einfacher/ } \\
\text { mittlerer } \\
\text { Dienst }\end{array}$} & Vater & 46,00 & 55,75 & 52,83 & 60,00 & Mutter & 38,00 & 49,00 & 59,50 & 69,00 \\
\hline & N1 & 4 & 8 & 6 & 2 & & 1 & 7 & 2 & 2 \\
\hline & N2 & 148 & 139 & 132 & 84 & & 152 & 153 & 148 & 58 \\
\hline
\end{tabular}


Fortsetzung Tabelle 1

\begin{tabular}{|c|c|c|c|c|c|c|c|c|c|c|}
\hline \multirow{3}{*}{$\begin{array}{c}08 \\
\text { gehobener } \\
\text { Dienst }\end{array}$} & Vater & 62,25 & 64,09 & 67,46 & 62,50 & Mutter & 66,00 & 69,00 & 64,00 & - \\
\hline & N1 & 8 & 11 & 11 & 2 & & 3 & 2 & 3 & 0 \\
\hline & N2 & 398 & 503 & 535 & 211 & & 403 & 455 & 509 & $167 X$ \\
\hline \multirow{3}{*}{$\begin{array}{c}09 \\
\text { höherer } \\
\text { Dienst }\end{array}$} & Vater & 77,00 & 79,50 & 74,67 & 77,00 & Mutter & - & 69,00 & 51,00 & - \\
\hline & N1 & 1 & 2 & 3 & 1 & & 0 & 1 & 1 & 0 \\
\hline & N2 & 511 & 622 & 794 & 357 & & $322 X$ & 386 & 499 & $203 X$ \\
\hline \multirow{3}{*}{$\begin{array}{c}10 \\
\text { kleinere Selb- } \\
\text { ständige }\end{array}$} & Vater & 57,33 & 52,14 & 51,00 & 56,00 & Mutter & 74,25 & 55,40 & 57,67 & - \\
\hline & N1 & 3 & 7 & 2 & 1 & & 4 & 5 & 3 & 0 \\
\hline & N2 & 420 & 515 & 581 & 301 & & 279 & 307 & 351 & $182 X$ \\
\hline \multirow{3}{*}{$\begin{array}{c}11 \\
\text { mittlere Selb- } \\
\text { ständige }\end{array}$} & Vater & 50,08 & 44,10 & 46,69 & 52,50 & Mutter & 51,00 & 52,00 & 49,00 & - \\
\hline & N1 & 12 & 10 & 13 & 4 & & 2 & 2 & 1 & 0 \\
\hline & N2 & 210 & 262 & 251 & 100 & & 66 & 77 & 92 & $36 X$ \\
\hline \multirow{3}{*}{$\begin{array}{c}12 \\
\text { größere Selb- } \\
\text { ständige }\end{array}$} & Vater & 65,00 & 59,16 & 58,57 & 47,00 & Mutter & - & - & 87,00 & - \\
\hline & N1 & 5 & 6 & 7 & 2 & & 0 & 0 & 1 & 0 \\
\hline & N2 & 62 & 78 & 84 & 40 & & $16 X$ & $18 X$ & 13 & $7 X$ \\
\hline \multirow{3}{*}{$\begin{array}{c}13 \\
\text { Freie Berufe, } \\
\text { selbst. Aka- } \\
\text { demiker/innen }\end{array}$} & Vater & 77,43 & 83,29 & 75,67 & 73,67 & Mutter & 69,50 & - & 88,00 & 88,00 \\
\hline & N1 & 7 & 7 & 3 & 6 & & 2 & 0 & 1 & 1 \\
\hline & N2 & 493 & 571 & 708 & 298 & & 324 & $359 x$ & 383 & 170 \\
\hline \multirow{3}{*}{$\begin{array}{l}14 \text { selbst- } \\
\text { ständige } \\
\text { Landwirte } \\
\text { /innen }\end{array}$} & Vater & 37,00 & 30,75 & 23,00 & 23,00 & Mutter & - & - & - & - \\
\hline & N1 & 4 & 4 & 2 & 1 & & 0 & 0 & 0 & 0 \\
\hline & N2 & 44 & 61 & 55 & 33 & & $18 x$ & $22 x$ & $32 x$ & $14 X$ \\
\hline
\end{tabular}

N1 = ALLBUS; N2 = Studierendensurvey 2000/01 - 2009/10, AG Hochschulforschung, Universität Konstanz $X=$ erzeugte fehlende Werte

\subsubsection{Elterliche Bildung}

Zusätzlich zum theoretisch zentralen SÖS wird der elterliche Bildungshintergrund unter einem explorativen Gesichtspunkt als zweite Dimension der sozialen Herkunft miteinbezogen. Im Querschnittmodell wird der höchste elterliche Bildungsabschluss (schulisch einschließlich Hochschulbildung) getrennt nach Vater und Mutter als unabhängige Variable in die Analysen integriert. Als abhängige Variable im Längsschnittmodell wird die elterliche Bildung hingegen in Form der durchschnittlichen Anzahl an Bildungsjahren (Dominance Model) linear auf den kategorial operationalisierten Exzellenzstatus regressiert. 


\section{Hypothesentests}

\subsection{Querschnittdesign: Determinanten der Wahl einer Exzellenzuniversität}

\subsubsection{Deskriptive Ergebnisse}

Tabelle 2 Verteilung der Studierendenmerkmale nach Exzellenzstatus der Universität zu Studienbeginn

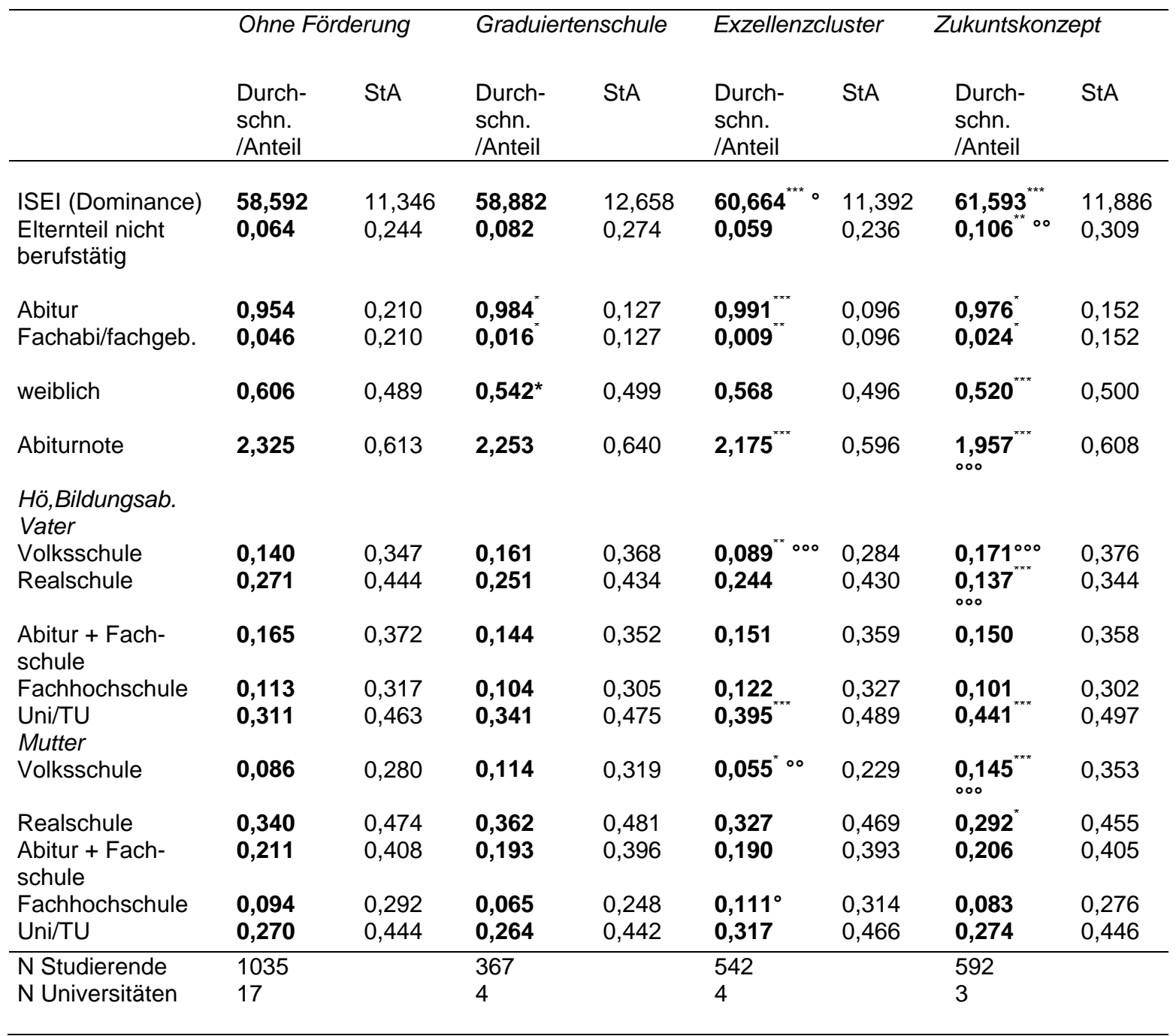

Signifikanz der Mittelwertdifferenzen gegenüber „ohne Exzellenzstatus: ${ }^{*} p<0,05,{ }^{\star *} p<0,01,{ }^{\star \star \star} p<0,001$; Signifikanz der Mittelwertdifferenz gegenüber der niedrigeren Förderlinie: ${ }^{\circ} p<0,05,{ }^{\circ} p<0,01,{ }^{\circ} p<0,001$

(Zukunftskonzept gegen Exzellenzcluster; Exzellenzcluster gegen Graduiertenschule);Wertebereiche ISEI: 16-90; Wertebereich Abiturnote: 1 (sehr gut) - 6 (ungenügend);

Stichprobe: Studierende, die ihr Studium nach Veröffentlichung der ersten Förderentscheidungen (Oktober 2006) begonnen haben; Quelle: Studierendensurvey 2009/10, AG Hochschulforschung, Universität Konstanz

Tabelle 2 zeigt die Verteilung individueller Merkmale von Studierenden differenziert nach Exzellenzstatus bzw. Förderlinie der Universität zu Studienbeginn. Die Stichprobe beinhaltet ausschließlich Studierende, die ihr Studium nach Veröffentlichung der ersten Förderent- 
scheidungen von Oktober 2006 oder 2007 (je nach Universität) begonnen haben. Die drei Gruppen, deren Universitäten zu Studienbeginn die jeweiligen Förderlinien innehatten, wurden auf signifikante Mittelwert-Abweichungen gegen die Gruppe getestet, die ihr Studium an einer Universität ohne Förderung begonnen hat. Studierende, die eine Universität mit Exzellenzcluster oder Zukunftskonzept gewählt haben, verfügen im Durchschnitt über einen hochsignifikant $(p<0,001)$ höheren ISEI (Wertebereich: 16 bis 90) als Studierende, die eine Universität ohne Förderung gewählt haben. Um Hierarchien zwischen den Universitäten nach Exzellenzstatus zu prüfen, wurden zusätzlich die Abweichungen zur jeweils niedrigeren Förderlinie auf Signifikanz getestet. Zentrales Ergebnis ist, dass die ISEI-Differenz der Kategorie Exzellenzcluster zwar zur Kategorie Graduiertenschule signifikant ist $(p<0,1)$, zwischen Zukunftskonzept- und Exzellenzcluster-Universitäten jedoch keine signifikante Differenz erkennbar ist.

Es fällt außerdem auf, dass in den beiden letztgenannten Gruppen hochsignifikant mehr Väter einen Universitätsabschluss besitzen als Studierende, die ihr Studium an einer nicht geförderten Universität aufgenommen haben. Des Weiteren besitzen in der Kategorie Zukunftskonzept hochsignifikant weniger Väter einen Realschulabschluss und in der Kategorie Exzellenzcluster signifikant ( $p>0,01$ ) weniger einen Volksschulabschluss.

Mütter hingegen, deren Kinder Zukunftskonzept-Universitäten gewählt haben, haben hochsignifikant häufiger einen Volksschulabschluss erlangt. Zudem zeichnen sich Studierende, die die Förderlinie Zukunftskonzept oder Exzellenzcluster gewählt haben durch einen hochsignifikant besseren Abiturnotendurchschnitt gegenüber der Kategorie „ohne Exzellenzstatus“ und „Exzellenzcluster" aus. Studienanfänger an Exzellenzcluster- und Zukunftskonzept-Universitäten verfügen zudem über einen hochsignifikant bzw. signifikant höheren Anteil an Abiturienten. 


\subsubsection{Multivariate Analysen}

Tabelle 3 Multinomiale logistische Regression. AV: Exzellenzstatus der Universität zu Studienbeginn. Odds Ratios

\begin{tabular}{|c|c|c|c|}
\hline & Graduiertenschule & $\begin{array}{l}\text { Exzellenz- } \\
\text { cluster }\end{array}$ & Zukunftskonzept \\
\hline ISEI (Dominance) & $\begin{array}{l}1,002 \\
(0,006)\end{array}$ & $\begin{array}{l}1,009 \\
(0,008)\end{array}$ & $\begin{array}{l}1,021^{* *} \\
(0,007)\end{array}$ \\
\hline Elternteil nicht berufstätig & $\begin{array}{l}1,257 \\
(0,334)\end{array}$ & $\begin{array}{l}0,969 \\
(0,387)\end{array}$ & $\begin{array}{l}1,553 \\
(0,435)\end{array}$ \\
\hline \multicolumn{4}{|l|}{$\begin{array}{l}\text { Hö,Bildungsab.Vater } \\
\text { (Ref.: Volksschule) }\end{array}$} \\
\hline - Realschule & $\begin{array}{l}0,807 \\
(0,430)\end{array}$ & $\begin{array}{l}1,234 \\
(0,579)\end{array}$ & $\begin{array}{l}0,425^{\pi \times} \\
(0,115)\end{array}$ \\
\hline - Abitur+Fachschule & $\begin{array}{l}0,811 \\
(0,166)\end{array}$ & $\begin{array}{l}1,246 \\
(0,298)\end{array}$ & $\begin{array}{l}0,756 \\
(0,177)\end{array}$ \\
\hline - Fachhochschule & $\begin{array}{l}0,842 \\
(0,236)\end{array}$ & $\begin{array}{l}1,246 \\
(0,393)\end{array}$ & $\begin{array}{l}0,584^{\pi} \\
(0,148)\end{array}$ \\
\hline - Uni/TU & $\begin{array}{l}0,992 \\
(0,287)\end{array}$ & $\begin{array}{l}1,419 \\
(0,428)\end{array}$ & $\begin{array}{l}0,955 \\
(0,205)\end{array}$ \\
\hline \multicolumn{4}{|l|}{$\begin{array}{l}\text { Hö,Bildungsab.Mutter } \\
\text { (Ref.: Volksschule) }\end{array}$} \\
\hline - Realschule & $\begin{array}{l}0,813 \\
(0,287)\end{array}$ & $\begin{array}{l}1,246 \\
(0,532)\end{array}$ & $\begin{array}{l}0,472^{\pi \times \pi} \\
(0,101)\end{array}$ \\
\hline - Abitur+Fachschule & $\begin{array}{l}0,676 \\
(0,232)\end{array}$ & $\begin{array}{l}1,067 \\
(0,411)\end{array}$ & $\begin{array}{l}0,446^{\star * \pi} \\
(0,108)\end{array}$ \\
\hline - Fachhochschule & $\begin{array}{l}0,502 \\
(0,239)\end{array}$ & $\begin{array}{l}1,293 \\
(0,816)\end{array}$ & $\begin{array}{l}0,386^{\times \varkappa} \\
(0,117)\end{array}$ \\
\hline - Uni/TU & $\begin{array}{l}0,646 \\
(0,354)\end{array}$ & $\begin{array}{l}1,169 \\
(0,602)\end{array}$ & $\begin{array}{l}0,291^{\pi \times \pi} \\
(0,072)\end{array}$ \\
\hline \multicolumn{4}{|l|}{$\begin{array}{l}\text { Studienfach } \\
\text { (Ref,: Soz,/ Kult.wiss.) }\end{array}$} \\
\hline - Jura & $\begin{array}{l}4,347^{\pi \pi} \\
(2,104)\end{array}$ & $\begin{array}{l}3,285^{\pi \pi} \\
(1,428)\end{array}$ & $\begin{array}{l}5,044^{\pi \pi \pi} \\
(2,141)\end{array}$ \\
\hline - Wirtschaftswiss. & $\begin{array}{l}0,517 \\
(0,205)\end{array}$ & $\begin{array}{l}1,071 \\
(0,330)\end{array}$ & $\begin{array}{l}1,428 \\
(0,938)\end{array}$ \\
\hline - Medizin & $\begin{array}{l}1,192 \\
(0,484)\end{array}$ & $\begin{array}{l}1,441 \\
(0,609)\end{array}$ & $\begin{array}{l}1,548 \\
(0,777)\end{array}$ \\
\hline - Naturwiss. & $\begin{array}{l}1,120 \\
(0,369)\end{array}$ & $\begin{array}{l}1,506 \\
(0,316)\end{array}$ & $\begin{array}{l}2,851^{\star \pi \times \pi} \\
(0,872)\end{array}$ \\
\hline - Ingenieurwiss. & $\begin{array}{l}1,131 \\
(0,740)\end{array}$ & $\begin{array}{l}2,435 \\
(1,866)\end{array}$ & $\begin{array}{l}2,058 \\
(2,301)\end{array}$ \\
\hline - andere & $\begin{array}{l}4,347^{x^{*}} \\
(2,104)\end{array}$ & $\begin{array}{l}0,982 \\
(0,176)\end{array}$ & $\begin{array}{l}0,748 \\
(0,167)\end{array}$ \\
\hline weiblich & $\begin{array}{l}0,728 \\
(0,149)\end{array}$ & $\begin{array}{l}0,982 \\
(0,176)\end{array}$ & $\begin{array}{l}0,748 \\
(0,167)\end{array}$ \\
\hline \multicolumn{4}{|l|}{$\begin{array}{l}\text { Hochschulreife } \\
\text { (Ref.: Abitur) }\end{array}$} \\
\hline Fachabi/fachgeb. & $\begin{array}{l}0,387 \\
(0,296)\end{array}$ & $\begin{array}{l}0,214 \\
(0,186)\end{array}$ & $\begin{array}{l}0,584 \\
(0,500)\end{array}$ \\
\hline Abiturnote & $\begin{array}{l}1,020 \\
(0,026)\end{array}$ & $\begin{array}{l}1,031 \\
(0,017)\end{array}$ & $\begin{array}{l}1,104^{\pi \times x} \\
(0,035)\end{array}$ \\
\hline $\begin{array}{l}\text { Pseudo } R^{2} \\
\mathrm{~N}\end{array}$ & $\begin{array}{l}0,063 \\
2536\end{array}$ & & \\
\hline
\end{tabular}

Referenz-Outcome: Universität erhält zu Studienbeginn keine Förderung; geclusterte Standardfehler in Klammern; ${ }^{*} p<0,05,{ }^{* *} p<0,01,{ }^{* * *} p<0,001$;

Stichprobe: Studierende, die ihr Studium nach Veröffentlichung der ersten Förderentscheidungen (Oktober 2006) begonnen haben; Quelle: Studierendensurvey 2009/10, AG Hochschulforschung, Universität Konstanz 
Tabelle 3 zeigt ein multinomiales logistisches Regressionsmodell auf die abhängige Variable „Exzellenzstatus der Universität zu Studienbeginn“ unter Einbezug derselben Stichprobe wie die deskriptiven Analysen aus Tabelle 2. Die Wahrscheinlichkeit, eine Universität mit Zukunftskonzept gegenüber einer Universität ohne Förderung durch die Exzellenzinitiative zu bevorzugen ist für Studierende mit hohem ISEI geringfügig $(2,1 \%)$, aber signifikant $(p<0,01)$ höher als für Studierende mit niedrigem ISEI. Dies gilt selbst bei statistischer Kontrolle unter anderem der Abiturnote und der erlangten Hochschulreife. Positive Odds Ratios des sozioökonomischen Status sind für eine Präferenz der übrigen Förderlinien zwar messbar, allerdings nicht gegen ein zufälliges Zustandekommen abgesichert. Unerwartet enthält die Kategorie Zukunftskonzept-Universitäten signifikant negative Odds Ratios höherer Bildungsabschlüsse gegenüber der Referenzkategorie Volksschulabschluss, welche im Fall der Mutter größtenteils hochsignifikant ausfallen. Auf den Versuch einer Erklärung soll an dieser Stelle verzichtet werden, da der SÖS das theoretische Konstrukt darstellt und zudem das Untersuchungsdesign, wie später noch gezeigt, keinen ausreichend validen Hypothesentest ermöglicht. Weiterhin ist in explorativer Hinsicht interessant, dass die Abiturnote unter Kontrolle der sozialen Herkunft die Präferenz einer Zukunftskonzept-Universität gegenüber einer Universität ohne Exzellenzstatus deutlich und signifikant wahrscheinlicher macht $(p<0,01)$. Die Schulleistung begünstigt also bei gleicher sozialer Herkunft die Wahl einer „Eliteuniversität“ der Förderlinie 3.

\subsection{Längsschnittdesign: Die Wirkung der Exzellenzinitiative auf die Hoch- schulwahl von Studierenden mit hohem SöS}

Eine signifikante Abhängigkeit der Hochschulwahl vom SÖS konnte lediglich in Bezug auf den Exzellenzstatus Zukunftskonzept festgestellt werden. Dieses Ergebnis scheint auf den ersten Blick eine Wirkung des Zukunftskonzepts als finanzintensivstem und prestigereichstem Exzellenzstatus auf die Attraktivität dieser Universitäten für Akteure hoher SÖS-Gruppen empirisch zu bestätigen. Um an dieser Stelle einen Kausalzusammenhang zwischen Exzellenzinitiative und Hochschulwahl folgern zu können, erweisen sich die Ergebnisse jedoch als gänzlich ungeeignet. Um dies zu verdeutlichen, soll genauer ausgeführt werden, was eine Ursache-/ Wirkungsbeziehung auszeichnet: Kausalität ist als reziprokes zeitliches Aufeinanderfolgen von Ereignissen bei gegebener Kontrafaktizität, also einem Nichteintritt der Folge bei Abwesenheit des Treatments definiert (Opp 2010), weshalb der Test einer Kausalhypothese am ehesten über eine Berücksichtigung dieser zentralen Bedingungen im Untersuchungsdesign erfolgen kann (s.a. Morgan und Winship 2010). Zu diesem Zweck prüft im Folgenden das Längsschnittmodell einen Effekt der Zuweisung eines Exzellenzstatus auf die sozioökonomische Zusammensetzung der Studierendenschaft einer Universität über die Zeit 
(Tabelle 4), während das multivariate Querschnittsmodell (Tabelle 3) nur einen rein korrelativen Zusammenhang zwischen Exzellenzinitiative und Universitätswahl in Abhängigkeit des SÖS abbildet. Neun Universitäten im Datensatz weisen Varianz im Exzellenzstatus über die Zeit auf. Drei Universitäten erhalten spätestens mit der zweiten der beiden Förderentscheidungen 2006 und 2007 ein Zukunftskonzept, vier mindestens ein Exzellenzcluster und zwei lediglich eine Graduiertenschule. Die technische Umsetzung des Fixed-Effects-Modells erfolgt neben einer Konstanthaltung zeitstabiler Universitätseffekte über eine Kontrolle der vier Messzeitpunkt-Effekte. Dies ist notwendig, weil aufgrund der sozialen Öffnung der Hochschulen im Zuge der Bildungsexpansion ein positiver Effekt der Zeit auch in Abwesenheit des Treatments vermutet wird, welcher bei nicht erfolgender Kontrolle die Schätzung des Kausaleffektes verzerren kann.

Tabelle 4 Fixed-Effects Regression (pooled OLS). AV: ISEI (Dominance - Model)

\begin{tabular}{|c|c|}
\hline Graduiertenschule & $\begin{array}{l}-0,934 \\
(0,494)\end{array}$ \\
\hline Exzellenzcluster & $\begin{array}{l}0,460 \\
(0,600)\end{array}$ \\
\hline Zukunftskonzept & $\begin{array}{l}-0,759 \\
(0,748)\end{array}$ \\
\hline TU Berlin & $\begin{array}{l}-1,718^{\star \star \star} \\
(0,0330)\end{array}$ \\
\hline Uni Bochum & $\begin{array}{l}-3,764^{\star * * x} \\
(0,0595)\end{array}$ \\
\hline TU Dresden & $\begin{array}{l}-4,007^{*+* x} \\
(0,0701)\end{array}$ \\
\hline Uni Frankfurt & $\begin{array}{l}-2,771^{\star \star *} \\
(0,0586)\end{array}$ \\
\hline Uni Freiburg & $\begin{array}{l}1,345^{\text {*ᄎ๙ }} \\
(0,0249)\end{array}$ \\
\hline Uni Hamburg & $\begin{array}{l}-1,091^{\star \star \star} \\
(0,0417)\end{array}$ \\
\hline Uni Karlsruhe & $\begin{array}{l}-1,260 \\
(0,0104)\end{array}$ \\
\hline Uni Leipzig & $\begin{array}{l}-3,102^{\star \star \star} \\
(0,056)\end{array}$ \\
\hline Welle 2000/01 & $\begin{array}{l}1,359 \\
(0,651)\end{array}$ \\
\hline Welle 2003/04 & $\begin{array}{l}-3,102^{\star \star \star} \\
(0,056)\end{array}$ \\
\hline Welle 2006/07 & $\begin{array}{l}1,359 \\
(0,651) \\
0,949\end{array}$ \\
\hline Constant & $\begin{array}{l}(0,505) \\
0,442\end{array}$ \\
\hline $\begin{array}{l}R^{2} \\
\mathrm{~N}\end{array}$ & $\begin{array}{l}0,021 \\
15783\end{array}$ \\
\hline
\end{tabular}

geclusterte Standardfehler in Klammern; ${ }^{*} p<0,05$, ${ }^{* \star} p<0,01$, ${ }^{\star \star \star} p<0,001$; Stichprobe: Studierende auf Universitäten, die ab 2006/2007 gefördert werden; Quelle: Studierendensurvey 2000/01 - 2009/10, AG Hochschulforschung, Universität Konstanz 
Tabelle 5 Fixed - Effects Regression (pooled OLS). AV: Bildungsjahre der Eltern (Dominance - Model)

\begin{tabular}{|c|c|c|}
\hline Graduiertenschule & $\begin{array}{l}-0,225 \\
(0,276)\end{array}$ & $\begin{array}{l}\text { Ref.: } \\
\text { Keine Förderung }\end{array}$ \\
\hline Exzellenzcluster & $\begin{array}{l}0,0255 \\
(0,169)\end{array}$ & \\
\hline Zukunftskonzept & $\begin{array}{l}-0,0478 \\
(0,139)\end{array}$ & \\
\hline TU Berlin & $\begin{array}{l}0,442^{\pi \times \pi} \\
(0,0114)\end{array}$ & $\begin{array}{l}\text { Ref.: } \\
\text { Unversität München }\end{array}$ \\
\hline Uni Bochum & $\begin{array}{l}-0,956 \\
(0,0439)\end{array}$ & \\
\hline TU Dresden & $\begin{array}{l}0,419 \\
(0,0179)\end{array}$ & \\
\hline Uni Frankfurt & $\begin{array}{l}-0,407^{* \times *} \\
(0,0163)\end{array}$ & \\
\hline Uni Freiburg & $\begin{array}{l}0,294^{\pi \times x^{\prime}} \\
(0,0115)\end{array}$ & \\
\hline Uni Hamburg & $\begin{array}{l}-0,144^{\pi \star \hbar} \\
(0,00962)\end{array}$ & \\
\hline Uni Karlsruhe & $\begin{array}{l}-0,111^{\times \pi \times} \\
(0,002)\end{array}$ & \\
\hline Uni Leipzig & $\begin{array}{l}0,464^{* * \star} \\
(0,026)\end{array}$ & \\
\hline Welle 2000/01 & $\begin{array}{l}-0,073 \\
(0,167)\end{array}$ & Ref.: Welle 2009/10 \\
\hline Welle 2003/04 & $\begin{array}{l}0,005 \\
(0,145)\end{array}$ & \\
\hline Welle 2006/07 & $\begin{array}{l}0,100 \\
(0,126)\end{array}$ & \\
\hline Constant & $\begin{array}{l}14,59^{\pi \times \pi} \\
(0,133)\end{array}$ & \\
\hline $\begin{array}{l}R^{2} \\
\mathrm{~N}\end{array}$ & $\begin{array}{l}0,018 \\
16010\end{array}$ & \\
\hline
\end{tabular}

geclusterte Standardfehler in Klammern

${ }^{*} \mathrm{p}<0,05,{ }^{* *} \mathrm{p}<0,01,{ }^{* * *} \mathrm{p}<0,001$

Stichprobe: Studierende auf Universitäten, die ab 2006/2007 gefördert werden; Quelle: Studierendensurvey 2000/01 - 2009/10, AG Hochschulforschung, Universität Konstanz

Wie aus Tabelle 4 hervorgeht, kann kein statistisch signifikanter Effekt der Zuweisung einer Förderlinie auf die SÖS-Zusammensetzung der Studierendenschaft einer Universität über die Zeit gemessen werden. Mit den elterlichen Bildungsjahren der Studierenden als metrischer abhängiger Variable wird analog verfahren und ebenfalls kein Effekt des Exzellenzstatus gefunden, der statistisch gegen Zufallsfehler abgesichert ist (Tabelle 5). Letztlich lässt dieses, als relativ valide eingeschätztes Untersuchungsdesign es nicht zu, einen Kausalmechanismus zwischen einer Determinante der sozialen Herkunft und der Wahl einer durch die Exzellenzinitiative geförderten Universität zu vertreten. Die Kausalhypo- 
these einer Wirkung der Exzellenzinitiative auf die Universitätswahl in Abhängigkeit des SÖS für die Förderlinie 3 wird demnach als falsifiziert angesehen.

\section{$7 \quad$ Zwischenfazit}

Streng genommen wäre der Forschungsprozess damit abgeschlossen, doch die Widersprüchlichkeit zwischen Quer- und Längsschnittergebnissen legen tiefergehende Analysen nahe: Während die Grundhypothese falsifiziert ist, zeigt das Querschnittdesign (Tabelle 3) einen signifikanten korrelativen Zusammenhang zwischen der Wahl einer ZukunftskonzeptUniversität bzw. Exzellenzuniversität und dem SÖS eines Akteurs. Es wird als wahrscheinlich angesehen, dass zeitstabile Universitätsmerkmale, die neben dem Exzellenzstatus unbeobachtet bleiben, die individuelle Hochschulwahl in Abhängigkeit des SÖS determinieren. Diese latenten Drittvariablen könnten ihre Wirkung auf die Hochschulwahl der Studierenden mit hohem SÖS über einen Prestigemechanismus entfalten, wobei das hochschuleigene Prestige bereits zu einem Zeitpunkt über die Qualität einer Universität informiert, zu dem die Förderentscheidungen der Exzellenzinitiative noch nicht öffentlich kommuniziert wurden. Es scheint nicht abwegig, dass die Förderentscheidungen der Exzellenzinitiative an solchen zeitstabilen vertikalen Differenzen zwischen Universitäten ausgerichtet sind, zumal sie auf qualitativen Evaluationen des universitätseigenen Potenzials beruhen, um überhaupt zu einer internationalen Spitzenuniversität gemacht werden zu können (vgl. Bundesministerium für Bildung und Forschung 2005). Diese Annahme ist mit der theoretischen Perspektive der SEU-Theorie (Erikson und Jonsson 1996, Breen und Goldthorpe 1997) prinzipiell vereinbar, solange der Exzellenzstatus als relevantes Universitätsmerkmal durch einen zeitstabilen Faktor ersetzt wird. Folglich soll im weiteren Verlauf dieses Beitrags geklärt werden, inwieweit die hier aufgezeigten, mit den Förderentscheidungen zwar korrelierenden, aber aus diesen nicht resultierenden SÖS-Selektionseffekte bereits vor dem Veröffentlichungszeitpunkt der Förderentscheidungen bestanden. Wäre dies der Fall, könnte es als Indiz gewertet werden, dass die Rolle der Exzellenzinitiative in einer nachträglichen Markierung und Förderung von SÖS-selektiven Universitäten besteht. Zusätzlich sollen Anhaltspunkte gesucht werden, ob hochschuleigene Prestigewirkungen eine SÖS-abhängige Hochschulwahl bewirken. 


\section{Test zeitstabiler SÖS-Selektionseffekte auf Zukunftskonzept- Universitäten}

Analog zum multinomialen logistischen Regressionsmodell mit Studierenden, die nach Veröffentlichung der Förderentscheidungen ihr Studium begonnen haben (Posttest-Modell) (Tabelle 3) wird an dieser Stelle ein äquivalentes Modell gerechnet, dessen abhängige Variable der zukünftige, durch die beiden Förderentscheidungen von 2006 und 2007 zugewiesene Exzellenzstatus der gewählten Universität ist. Einbezogen werden im Gegensatz zur Stichprobe des Posttest-Modells diejenigen Studierenden, die ihr Studium vor Veröffentlichung der Förderentscheidungen (Pretest-Modell) begonnen haben. Es basiert auf einer gepoolten Stichprobe der Wellen 2000/01, 2003/04 und 2006/07.

Tabelle 6 Pretest: Multinomiale logistische Regression: AV: Zukünftiger Exzellenzstatus der gewählten Universität. Odds Ratios; Posttest: siehe Tabelle 3

\begin{tabular}{|c|c|c|c|c|c|c|}
\hline & \multicolumn{2}{|c|}{ Graduiertenschule } & \multicolumn{2}{|c|}{ Exzellenzcluster } & \multicolumn{2}{|c|}{ Zukunftskonzept } \\
\hline & Pretest & $\begin{array}{l}\text { Posttest } \\
\text { (Tab. 3) }\end{array}$ & Pretest & $\begin{array}{l}\text { Posttest } \\
\text { (Tab. 3) }\end{array}$ & Pretest & $\begin{array}{l}\text { Posttest } \\
\text { (Tab. 3) }\end{array}$ \\
\hline ISEI (Dominance) & $\begin{array}{l}0,994 \\
(0,010)\end{array}$ & $\begin{array}{l}1,002 \\
(0,006)\end{array}$ & $\begin{array}{l}1,001 \\
(0,010)\end{array}$ & $\begin{array}{l}1,009 \\
(0,008)\end{array}$ & $\begin{array}{l}1,022^{\approx \pi x} \\
(0,006)\end{array}$ & $\begin{array}{l}1,021^{\pi \times} \\
(0,007)\end{array}$ \\
\hline $\begin{array}{l}\text { Elternteil nicht berufstä- } \\
\text { tig }\end{array}$ & $\begin{array}{l}0,902 \\
(0,345)\end{array}$ & $\begin{array}{l}1,257 \\
(0,334)\end{array}$ & $\begin{array}{l}0,908 \\
(0,251)\end{array}$ & $\begin{array}{l}0,969 \\
(0,387)\end{array}$ & $\begin{array}{l}1,342 \\
(0,247)\end{array}$ & $\begin{array}{l}1,553 \\
(0,435)\end{array}$ \\
\hline \multicolumn{7}{|l|}{$\begin{array}{l}\text { Hö,Bildungsab.Vater } \\
\text { (Ref.: Volksschule) }\end{array}$} \\
\hline - Realschule & $\begin{array}{l}1,144 \\
(0,641)\end{array}$ & $\begin{array}{l}0,807 \\
(0,430)\end{array}$ & $\begin{array}{l}1,125 \\
(0,381)\end{array}$ & $\begin{array}{l}1,234 \\
(0,579)\end{array}$ & $\begin{array}{l}0,493^{\pi \pi} \\
(0,120)\end{array}$ & $\begin{array}{l}0,425^{\star \pi} \\
(0,115)\end{array}$ \\
\hline - Abitur+Fachschule & $\begin{array}{l}1,214 \\
(0,281)\end{array}$ & $\begin{array}{l}0,811 \\
(0,166)\end{array}$ & $\begin{array}{l}1,198 \\
(0,203)\end{array}$ & $\begin{array}{l}1,246 \\
(0,298)\end{array}$ & $\begin{array}{l}0,922 \\
(0,132)\end{array}$ & $\begin{array}{l}0,756 \\
(0,177)\end{array}$ \\
\hline - Fachhochschule & $\begin{array}{l}1,178 \\
(0,511)\end{array}$ & $\begin{array}{l}0,842 \\
(0,236)\end{array}$ & $\begin{array}{l}1,045 \\
(0,237)\end{array}$ & $\begin{array}{l}1,246 \\
(0,393)\end{array}$ & $\begin{array}{l}0,830 \\
(0,145)\end{array}$ & $\begin{array}{l}0,584^{* \prime} \\
(0,148)\end{array}$ \\
\hline - Uni/TU & $\begin{array}{l}1,276 \\
(0,583)\end{array}$ & $\begin{array}{l}0,992 \\
(0,287)\end{array}$ & $\begin{array}{l}1,459 \\
(0,384)\end{array}$ & $\begin{array}{l}1,419 \\
(0,428)\end{array}$ & $\begin{array}{l}0,978 \\
(0,199)\end{array}$ & $\begin{array}{l}0,955 \\
(0,205)\end{array}$ \\
\hline \multicolumn{7}{|l|}{$\begin{array}{l}\text { Hö,Bildungsab.Mutter } \\
\text { (Ref.: Volksschule) }\end{array}$} \\
\hline - Realschule & $\begin{array}{l}0,960 \\
(0,349)\end{array}$ & $\begin{array}{l}0,813 \\
(0,287)\end{array}$ & $\begin{array}{l}1,173 \\
(0,322)\end{array}$ & $\begin{array}{l}1,246 \\
(0,532)\end{array}$ & $\begin{array}{l}0,628^{\times} \\
(0,142)\end{array}$ & $\begin{array}{l}0,472^{\pi \times \pi} \\
(0,101)\end{array}$ \\
\hline - Abitur+Fachschule & $\begin{array}{l}1,137 \\
(0,438)\end{array}$ & $\begin{array}{l}0,676 \\
(0,232)\end{array}$ & $\begin{array}{l}1,237 \\
(0,324)\end{array}$ & $\begin{array}{l}1,067 \\
(0,411)\end{array}$ & $\begin{array}{l}0,694^{\star \prime} \\
(0,128)\end{array}$ & $\begin{array}{l}0,446 \\
(0,108)\end{array}$ \\
\hline - Fachhochschule & $\begin{array}{l}1,033 \\
(0,681)\end{array}$ & $\begin{array}{l}0,502 \\
(0,239)\end{array}$ & $\begin{array}{l}1,105 \\
(0,554)\end{array}$ & $\begin{array}{l}1,293 \\
(0,816)\end{array}$ & $\begin{array}{l}0,410 \\
(0,134)\end{array}$ & $\begin{array}{l}0,386^{x t} \\
(0,117)\end{array}$ \\
\hline - Uni/TU & $\begin{array}{l}1,057 \\
(0,608)\end{array}$ & $\begin{array}{l}0,646 \\
(0,354)\end{array}$ & $\begin{array}{l}1,192 \\
(0,512)\end{array}$ & $\begin{array}{l}1,169 \\
(0,602)\end{array}$ & $\begin{array}{l}0,513^{x^{\prime}} \\
(0,151)\end{array}$ & $\begin{array}{l}0,291^{\star \star \pi} \\
(0,072)\end{array}$ \\
\hline $\begin{array}{l}\text { Studienfach } \\
\text { (Ref.: Soz,/ Kult, wiss,) }\end{array}$ & & & & & & \\
\hline - Jura & $\begin{array}{l}1,685 \\
(0,899)\end{array}$ & $\begin{array}{l}4,347^{\star \pi} \\
(2,104)\end{array}$ & $\begin{array}{l}1,905 \\
(0,918)\end{array}$ & $\begin{array}{l}3,285^{\star *} \\
(1,428)\end{array}$ & $\begin{array}{l}1,650 \\
(0,770)\end{array}$ & $\begin{array}{l}5,044^{\star \star \pi} \\
(2,141)\end{array}$ \\
\hline
\end{tabular}




\section{Fortsetzung Tabelle 6}

\begin{tabular}{|c|c|c|c|c|c|c|}
\hline - Wirtschaftswiss. & $\begin{array}{l}0,497^{\pi} \\
(0,140)\end{array}$ & $\begin{array}{l}0,517 \\
(0,205)\end{array}$ & $\begin{array}{l}0,873 \\
(0,218)\end{array}$ & $\begin{array}{l}1,071 \\
(0,330)\end{array}$ & $\begin{array}{l}0,724 \\
(0,350)\end{array}$ & $\begin{array}{l}1,428 \\
(0,938)\end{array}$ \\
\hline - Medizin & $\begin{array}{l}1,494 \\
(0,626)\end{array}$ & $\begin{array}{l}1,192 \\
(0,484)\end{array}$ & $\begin{array}{l}1,125 \\
(0,510)\end{array}$ & $\begin{array}{l}1,441 \\
(0,609)\end{array}$ & $\begin{array}{l}1,447 \\
(0,655)\end{array}$ & $\begin{array}{l}1,548 \\
(0,777)\end{array}$ \\
\hline - Naturwiss. & $\begin{array}{l}0,567 \\
(0,189)\end{array}$ & $\begin{array}{l}1,120 \\
(0,369)\end{array}$ & $\begin{array}{l}0,948 \\
(0,239)\end{array}$ & $\begin{array}{l}1,506 \\
(0,316)\end{array}$ & $\begin{array}{l}1,172 \\
(0,461)\end{array}$ & $\begin{array}{l}2,851 \times 1 \times \pi \\
(0,872)\end{array}$ \\
\hline - Ingenieurwiss. & $\begin{array}{l}0,340 \\
(0,298)\end{array}$ & $\begin{array}{l}1,131 \\
(0,740)\end{array}$ & $\begin{array}{l}1,779 \\
(1,353)\end{array}$ & $\begin{array}{l}2,435 \\
(1,866)\end{array}$ & $\begin{array}{l}1,025 \\
(1,100)\end{array}$ & $\begin{array}{l}2,058 \\
(2,301)\end{array}$ \\
\hline - andere & $\begin{array}{l}0,128 \\
(0,050)\end{array}$ & $\begin{array}{l}4,347^{* *} \\
(2,104)\end{array}$ & $\begin{array}{l}1,133 \\
(0,576)\end{array}$ & $\begin{array}{l}0,982 \\
(0,176)\end{array}$ & $\begin{array}{l}0,515 \\
(0,303)\end{array}$ & $\begin{array}{l}0,748 \\
(0,167)\end{array}$ \\
\hline weiblich & $\begin{array}{l}0,930 \\
(0,051)\end{array}$ & $\begin{array}{l}0,728 \\
(0,149)\end{array}$ & $\begin{array}{l}0,899 \\
(0,047)\end{array}$ & $\begin{array}{l}0,982 \\
(0,176)\end{array}$ & $\begin{array}{l}0,740 \\
(0,128)\end{array}$ & $\begin{array}{l}0,748 \\
(0,167)\end{array}$ \\
\hline \multicolumn{7}{|l|}{$\begin{array}{l}\text { Hochschulreife } \\
\text { (Ref,: Abitur) }\end{array}$} \\
\hline - Fachabi/fachgeb. & $\begin{array}{l}0,258 \times \\
(0,118)\end{array}$ & $\begin{array}{l}0,387 \\
(0,296)\end{array}$ & $\begin{array}{l}0,230 \\
(0,116)\end{array}$ & $\begin{array}{l}0,214 \\
(0,186)\end{array}$ & $\begin{array}{l}0,409 \\
(0,199)\end{array}$ & $\begin{array}{l}0,584 \\
(0,500)\end{array}$ \\
\hline Abiturnote & $\begin{array}{l}1,021 \\
(0,036)\end{array}$ & $\begin{array}{l}1,020 \\
(0,026)\end{array}$ & $\begin{array}{l}1,013 \\
(0,017)\end{array}$ & $\begin{array}{l}1,031 \\
(0,017)\end{array}$ & $\begin{array}{l}1,068^{x \neq} \\
(0,022)\end{array}$ & $\begin{array}{l}1,104^{x} \\
(0,035)\end{array}$ \\
\hline $\begin{array}{l}\text { Pseudo } R^{2} \\
\mathrm{~N}\end{array}$ & $\begin{array}{l}0,047 \\
19415\end{array}$ & $\begin{array}{l}0,063 \\
2536\end{array}$ & & & & \\
\hline
\end{tabular}

Referenz - Outcome Pretest: Universität gewählt, die zukünftig nicht gefördert wird;

Referenz - Outcome Posttest: Universität erhält zu Studienbeginn keine Förderung (siehe Tab. 3);

geclusterte Standardfehler in Klammern; ${ }^{*} p<0,05,{ }^{* *} p<0,01,{ }^{* * *} p<0,001$;

Stichprobe Pretest: Studierende, die ihr Studium vor Veröffentlichung der ersten Förderentscheidungen (Oktober 2006) begonnen haben; Quelle: Studierendensurvey 2000/01 - 2009/10 AG Hochschulforschung, Universität Konstanz

Stichprobe Posttest: Studierende, die ihr Studium nach Veröffentlichung der ersten Förderentscheidungen (Oktober 2006) begonnen haben (siehe Tab. 3); Quelle: Studierendensurvey 2009/10, AG Hochschulforschung, Universität Konstanz

Ein Vergleich der beiden Querschnittmodelle (Tabelle 6) lässt ein prinzipiell deckungsgleiches Muster erkennen: Das Pretest-Modell zeigt unter Einbezug der gleichen Kontrollvariablen nahezu identische, signifikante positive Odds Ratios für die Förderlinie „Zukunftskonzept“ gegenüber dem Referenz-Outcome „keine Förderung“, während die der übrigen Förderlinien nicht signifikant ausfallen. Die Signifikanz der ISEI-Odds Ratios ist im Vergleich zum Posttest-Modell sogar hochsignifikant $(p<0,001)$. Der negative Einfluss der elterlichen Bildungsabschlüsse besteht auch in diesem Modell, jedoch fallen die entsprechenden Odds Ratios trotz weitaus höherer Fallzahlen weniger signifikant aus. Des Weiteren ist auch der im Posttest-Modell gefundene Selektionsmechanismus, der allein von der Abiturnote der Studierenden getragen wird, bei gleichem Signifikanzniveau zeitlich stabil und somit unabhängig von der Exzellenzinitiative. Außerdem interessant sind die signifikant negativen Odds Ratios $(p<0,01)$ für die Präferenz der Exzellenzcluster- und Graduiertenschule-Universitäten von Studierenden mit Fachabitur oder fachgebundener Hochschulreife gegenüber Studierenden mit Allgemeiner Hochschulreife im Pretest-Modell. Es kann zusammengefasst werden, dass sich die SÖS-Selektionseffekte auf Zukunftskonzept-Universitäten als zeitstabil darstellen, 
was die kausale Unabhängigkeit der SÖS-Selektionseffekte von der Exzellenzinitiative nahelegt und darüber hinaus für eine zeitliche Reaktion der Exzellenzinitiative auf konstante vertikale Differenzierungen im Hochschulsystem spricht.

\section{Test einer Prestigewirkung der SöS-selektiven Zukunftskonzept- Universitäten}

Es wird vermutet, dass der zuvor als zeitstabil nachgewiesene SÖS-Selektionseffekt auf Zukunftskonzept-Universitäten mit einer ebenso zeitstabilen höheren Prestigewahrnehmung dieser Universitäten durch die Studierenden einhergeht.

Um dies zu testen, werden retrospektive Angaben der Studierenden über das Ausmaß, in dem Ruf und Tradition der Universität für ihre Hochschulwahl eine Rolle spielten, als Indikator für die universitätsspezifische Prestigewirkung herangezogen. Weil Daten für diese Variable ausschließlich in der Welle 2006/2007, also vor Veröffentlichung der Förderentscheidung vorhanden sind, bleibt ein Test der zeitstabilen Prestigewirkung bzw. die Stichprobe des Regressionsmodells auf diesen Messzeitpunkt begrenzt. Mit Ausnahme der am Fuß der Tabelle integrierten Variable „Hochschulwahlmotiv Ruf und Tradition“ bleibt die Spezifikation des Modells mit den vorherigen Querschnittanalysen identisch.

Das Modell ist in Tabelle 7 dargestellt: Studierende die angeben, ihre Universität verstärkt aufgrund des organisationsspezifischen Prestiges gewählt zu haben, haben gegenüber der Gruppe mit wenig ausgeprägter Prestigeorientierung im Hochschulwahlmotiv eine mehr als $50 \%$ und hochsignifikant $(p<0,001)$ höhere Wahrscheinlichkeit, eine Universität, die später über die Förderlinie Zukunftskonzept gefördert wird, gegenüber einer Universität ohne zukünftige Förderung zu bevorzugen.

Die Integration der Variable in das Modell scheint nicht die bereits als zeitstabil herausgestellten signifikant positiven ISEI-Odds Ratios für die Präferenz einer ZukunftskonzeptUniversität zu erklären. Dies spricht dafür, dass zwischen den unterschiedlichen SÖSGruppen an Zukunftskonzept-Universitäten kein Unterschied in der Prestigewahrnehmung ihrer Universität besteht, was mit der SEU-Theorie (Erikson und Jonsson 1996, Breen und Goldthorpe 1997) grundsätzlich in Einklang gebracht werden kann. Die Ergebnisse zeigen, dass die zeitstabilen SÖS-Selektionseffekte auf Zukunftskonzept-Universitäten mit einer höheren Prestigewirkung dieser Universitäten auf ihre Studierenden einhergehen. 
Tabelle 7 Multinomiale logistische Regression: AV: Zukünftiger Exzellenzstatus der gewählten Universität. Odds Ratios

\begin{tabular}{|c|c|c|c|}
\hline & Graduiertenschule & Exzellenzcluster & ZukunftsKonzept \\
\hline ISEI (Dominance) & $\begin{array}{l}0,991 \\
(0,012)\end{array}$ & $\begin{array}{l}0,995 \\
(0,008)\end{array}$ & $\begin{array}{l}1,023^{\star \pi} \\
(0,007)\end{array}$ \\
\hline Elternteil nicht berufstätig & $\begin{array}{l}1,019 \\
(0,376)\end{array}$ & $\begin{array}{l}0,799 \\
(0,245)\end{array}$ & $\begin{array}{l}1,543 \\
(0,344)\end{array}$ \\
\hline \multicolumn{4}{|l|}{$\begin{array}{l}\text { Hö,Bildungsab.Vater } \\
\text { (Ref.: Volksschule) }\end{array}$} \\
\hline - Realschule & $\begin{array}{l}1,336 \\
(0,691)\end{array}$ & $\begin{array}{l}1,041 \\
(0,406)\end{array}$ & $\begin{array}{l}0,386^{\pi \pi \pi} \\
(0,102)\end{array}$ \\
\hline - Abitur+Fachschule & $\begin{array}{l}1,340 \\
(0,296)\end{array}$ & $\begin{array}{l}1,155 \\
(0,256)\end{array}$ & $\begin{array}{l}0,763 \\
(0,155)\end{array}$ \\
\hline - Fachhochschule & $\begin{array}{l}1,489 \\
(0,656)\end{array}$ & $\begin{array}{l}1,022 \\
(0,245)\end{array}$ & $\begin{array}{l}0,740 \\
(0,147)\end{array}$ \\
\hline - Uni/TU & $\begin{array}{l}1,248 \\
(0,521)\end{array}$ & $\begin{array}{l}1,434 \\
(0,391)\end{array}$ & $\begin{array}{l}0,761 \\
(0,156)\end{array}$ \\
\hline \multicolumn{4}{|l|}{$\begin{array}{l}\text { Hö.Bildungsab.Mutter } \\
\text { (Re.: Volksschule) }\end{array}$} \\
\hline - Realschule & $\begin{array}{l}1,021 \\
(0,328)\end{array}$ & $\begin{array}{l}1,409 \\
(0,380)\end{array}$ & $\begin{array}{l}0,604^{*} \\
(0,145)\end{array}$ \\
\hline - Abitur+Fachschule & $\begin{array}{l}1,251 \\
(0,307)\end{array}$ & $\begin{array}{l}1,555^{x^{\prime \prime}} \\
(0,309)\end{array}$ & $\begin{array}{l}0,681 \\
(0,147)\end{array}$ \\
\hline - Fachhochschule & $\begin{array}{l}1,003 \\
(0,621)\end{array}$ & $\begin{array}{l}1,357 \\
(0,596)\end{array}$ & $\begin{array}{l}0,484^{*} \\
(0,165)\end{array}$ \\
\hline - Uni/TU & $\begin{array}{l}1,129 \\
(0,592)\end{array}$ & $\begin{array}{l}1,383 \\
(0,490)\end{array}$ & $\begin{array}{l}0,531^{\star \prime} \\
(0,149)\end{array}$ \\
\hline \multicolumn{4}{|l|}{$\begin{array}{l}\text { Studienfach } \\
\text { (Ref.: Soz,/ Kult,wiss,) }\end{array}$} \\
\hline - Jura & $\begin{array}{l}2,774 \\
(1,866)\end{array}$ & $\begin{array}{l}3,780^{\pi} \\
(2,542)\end{array}$ & $\begin{array}{l}3,341 \\
(2,192)\end{array}$ \\
\hline - Wirtschaftswiss. & $\begin{array}{l}0,467 \\
(0,184)\end{array}$ & $\begin{array}{l}0,761 \\
(0,184)\end{array}$ & $\begin{array}{l}0,613 \\
(0,289)\end{array}$ \\
\hline - Medizin & $\begin{array}{l}1,659 \\
(0,925)\end{array}$ & $\begin{array}{l}1,728 \\
(1,005)\end{array}$ & $\begin{array}{l}1,777 \\
(1,130)\end{array}$ \\
\hline - Naturwiss. & $\begin{array}{l}0,563 \\
(0,197)\end{array}$ & $\begin{array}{l}0,850 \\
(0,260)\end{array}$ & $\begin{array}{l}0,982 \\
(0,413)\end{array}$ \\
\hline - Ingenieurwiss. & $\begin{array}{l}0,249 \\
(0,268)\end{array}$ & $\begin{array}{l}1,557 \\
(1,218)\end{array}$ & $\begin{array}{l}0,755 \\
(0,835)\end{array}$ \\
\hline - andere & $\begin{array}{l}0,0541^{\pi x \times} \\
(0,025)\end{array}$ & $\begin{array}{l}1,196 \\
(0,765)\end{array}$ & $\begin{array}{l}0,370 \\
(0,233)\end{array}$ \\
\hline weiblich & $\begin{array}{l}0,758^{\times \pi x} \\
(0,052)\end{array}$ & $\begin{array}{l}0,827^{\prime \prime} \\
(0,072)\end{array}$ & $\begin{array}{l}0,744 \\
(0,130)\end{array}$ \\
\hline $\begin{array}{l}\text { Hochschulreife } \\
\text { (Ref.: Abitur) }\end{array}$ & & & \\
\hline - Fachabi/fachgeb. & $\begin{array}{l}0,156^{\star \star \star} \\
(0,069)\end{array}$ & $\begin{array}{l}0,0847^{* * *} \\
(0,056)\end{array}$ & $\begin{array}{l}0,239^{\star * *} \\
(0,103)\end{array}$ \\
\hline Abiturnote & $\begin{array}{l}1,030 \\
(0,034)\end{array}$ & $\begin{array}{l}1,019 \\
(0,017)\end{array}$ & $\begin{array}{l}1,063^{\pi x} \\
(0,021)\end{array}$ \\
\hline $\begin{array}{l}\text { Hochschulwahlmotiv: Ruf und } \\
\text { Tradition }\end{array}$ & $\begin{array}{l}1,040 \\
(0,078)\end{array}$ & $\begin{array}{l}1,035 \\
(0,101)\end{array}$ & $\begin{array}{l}1,544^{* * *} \\
(0,064)\end{array}$ \\
\hline $\begin{array}{l}\text { Pseudo } R^{2} \\
N\end{array}$ & $\begin{array}{l}0,095 \\
5617\end{array}$ & & \\
\hline
\end{tabular}

Referenz-Outcome: Universität gewählt, die zukünftig nicht gefördert wird; geclusterte Standardfehler in Klammern; ${ }^{*} p<0,05,{ }^{* *} p<0,01,{ }^{* * *} p<0,001$; Stichprobe: Studierende, die ihr Studium vor Veröffentlichung der ersten Förderentscheidungen (Oktober 2006) begonnen haben; Quelle: Studierendensurvey 2006/07, AG Hochschulforschung, Universität Konstanz 


\section{Einschätzung der Ergebnisse}

Aus methodischer Perspektive ist die Widersprüchlichkeit der Resultate aus Längs- und Querschnittsdesign nicht verwunderlich. Sie dient in diesem Fall gar dem Erkenntnisgewinn bezüglich der Rolle der Exzellenzinitiative im SÖS-Selektionsprozess und zeigt, dass Querschnittdaten nur begrenzt als Grundlage für Kausalschlüsse geeignet sind, selbst wenn mittels multivariater Regressionsanalysen für Effekte vermuteter Kovariate kontrolliert wird. Dennoch darf nicht unerwähnt bleiben, dass die Anwendung des Fixed-Effects-Modells zwar eine erhöhte Validität im Hypothesentest gewährleistet, die Gefahr des $\beta$-Fehlers jedoch unvermeidlich erhöht: Der Within-Schätzer begrenzt die zur Effektschätzung genutzte statistische Varianz zwischen Studierenden auf die Universitätsebene, was einen Verlust statistischer „Power" zur Folge hat und tendenziell positive Signifikanztests verhindert. Auch die Zuspielung der benötigten ISEI-Mittelwerte aus dem ALLBUS-Datensatz führt zu einem Informationsverlust mit der Folge, dass ISEI-Varianz zwischen Studierenden für die Effektschätzung ungenutzt bleibt.

Unter diesen Einschränkungen lautet der zentrale Befund dieses Beitrags, dass Studierende mit hohem SÖS ihre Hochschulwahl nicht am Exzellenzstatus einer Universität ausrichten, sondern eher am zeitlich stabilen Prestige einer Universität. Die Auszeichnung und finanzielle Förderung sogenannter „Exzellenzuniversitäten“ stellt sich als eine zeitliche Reaktion auf Universitätsmerkmale dar, welche mit bereits existierenden Prestigewirkungen auf die Studierenden korrelieren. Unter der Voraussetzung, dass Prestigedifferenzen tatsächliche Qualitätsunterschiede zwischen Universitäten widerspiegeln, hat die Exzellenzinitiative damit ihr Ziel erreicht, nämlich Spitzenuniversitäten zu identifizieren und sie zu markieren (vgl. Bundesministerium für Bildung und Forschung 2005). Die Ergebnisse sprechen allerdings auch dafür, dass der Exzellenzstatus neben zeitstabilen Prestigeunterschieden, vermutlich ungewollt, auch zeitstabile soziale Ungleichheit der Studierendenschaften zwischen Universitäten abbildet.

Obwohl aufgrund nicht erfolgter Messung der SEU-Entscheidungsdeterminanten kein umfassender Theorietest erfolgt, kann prinzipiell an die Erklärung der Ursprungshypothese angeknüpft werden. Hierzu muss das Universitätsmerkmal, welches die Attraktivität der Universität für hohe SÖS-Gruppen fördert, durch zeitstabiles Organisationsprestige ersetzt werden. Implikationen eines durch die Akteure angestrebten Statuserhalts über eine antizipierte Signalwirkung auf den Arbeitgeber scheinen weiterhin plausibel.

Nimmt man an, dass Prestigewirkungen gar objektive Qualitätsunterschiede abbilden, bedürfen hohe SÖS-Gruppen offensichtlich nicht erst der Sichtbarmachung bestehender Differenzierungen zwischen Universitäten durch die Exzellenzinitiative, um eine rationale Entscheidung treffen zu können. Sie scheinen bereits über zeitstabile Prestige- bzw. Quali- 
tätsunterschiede informiert zu sein, denn sie richten ihre Hochschulwahl signifikant daran aus. Dies lässt ihre Handlungen rationaler erscheinen als zunächst vermutet. Ob die SÖSSelektion auf prestigereiche Universitäten allerdings tatsächlich bessere Berufschancen dieser Gruppe auf dem Arbeitsmarkt und damit eine Reproduktion sozialer Ungleichheit über ein binnendifferenziertes Universitätssystem zur Folge hat, bleibt unklar. Hierzu bedarf es einer Untersuchung der Bildungsrenditen universitätsspezifischer Hochschulzertifikate.

Für die Zukunft ist auch die Untersuchung langfristiger Wirkungen der Eexzellenzinitiative sowie einer Wirkung der zweiten Programmphase interessant: Es ist denkbar, dass eine an Qualitätsdeterminanten ausgerichtete strukturelle Förderung von Universitäten durch eine Ausweitung tatsächlicher Qualitätsunterschiede eine Verstärkung der in dieser Arbeit gemessenen zeitstabilen Prestigeunterschiede zur Folge hat. Sollten sich hohe SÖS-Gruppen an diesen Differenzen orientieren, worauf die Ergebnisse dieser Untersuchung hindeuten, könnte dies eine Verstärkung der gefundenen SÖS-Selektionseffekte nach sich ziehen.

Zusätzlich zu diesen signifikanten zeitstabilen SÖS-Selektionseffekten zeichnet sich ein von der sozialen Herkunft unabhängiger Selektionsmechanismus nach ehemaligen Schulleistungen sowie der Art der erlangten Hochschulreife (hier Abitur) auf die prestigereichen Zukunftskonzept-Universitäten ab. Bei gleicher sozialer Herkunft erhöht die Abitur- bzw. Abschlussnote die Chance, eine Universität zu besuchen, welche gegebenenfalls über eine gehobene Ausbildungsqualität verfügt und deren Bildungszertifikat später eventuell besonders wertvoll ist. Dieser Schulleistungsmechanismus sowie die signifikanten negativen Bildungseffekte der Mutter weisen eher auf eine soziale Öffnungs- als Schließungstendenz prestigereicher Zukunftskonzept-Universitäten hin, bedürfen jedoch neben einer theoretischen Erklärung weiterer empirischer Befunde. 


\section{Literaturverzeichnis}

Allmendinger, Jutta (1989). Educational Systems and Labour Market Income. In: European Sociological Review, H.5, S.231-250

Arbeitsgruppe Hochschulforschung der Universität Konstanz (2007). Soziale Ungleichheit im Hochschulwesen. Barrieren für Bildungsaufsteiger. In: Tino Bargel (Hg.), Hefte zur Bildungs- und Hochschulforschung, H.49, S.1-40

Astin, Alexander W. \& Osegura, Leticia (2004). The Declining "Equity" of American Higher Education. In: The Review of Higher Education, H.27, S.321-341

Baker, Sally \& Brown, Brian (2007). Images of Excellence. Constructions of Institutional Prestige and Reflections in the University Choice Process. In: British Journal of Sociology of Education, H.28, S.377-391

Bastedo, Michael N. \& Bowman, Nicholas. A. (2010). U.S. News \& World Report College Rankings. Modeling Institutional Effects on Organizational Reputation. In: American Journal of Education, H.116, S.163-183

Becker, Gary S. (1964). Human Capital. A Theoretical and Empirical Analysis with Special Reference to Education. New York: Columbia University Press

Becker, Rolf \& Hecken, Anna E. (2007). Studium oder Berufsausbildung? Eine empirische Überprüfung der Modelle zur Erklärung von Bildungsentscheidungen von Esser sowie von Breen und Goldthorpe. In: Zeitschrift für Soziologie, H.36, S.100-117

Bowen, William. G. \& Bok, Derek (1998). The Shape of the River. New Jersey: Princeton University Press

Brüderl, Josef (2010). Kausalanalyse mit Paneldaten. In: Christof Wolf \& Henning Best (Hg.), Handbuch der sozialwissenschaftlichen Datenanalyse. Wiesbaden: VS Verlag. S. 963994

Büttner, Thies \& Kraus, Margit, Rincke, Johannes (2003). Hochschulranglisten als Qualitätsindikatoren im Wettbewerb der Hochschulen. In: Vierteljahreshefte zur Wirtschaftsforschung, H.72, S.252-270

Breen, Richard \& Goldthorpe, John. H. (1997). Explaining Educational Differentials. In: Rationality and Society, H.9, S.275-305

Brewer, Dominic \& Eide, Eric J., Ehrenberg, Ronald G.(1999). Does It Pay to Attend an Elite Private College? Cross-Cohort Evidence on the Effects of College Type on Earning. In: The Journal of Human Resources, H.34, S.104-123

Bundesministerium für Bildung und Forschung (2005). Bund-Länder-Vereinbarung gemäß Artikel $91 \mathrm{~b}$ des Grundgesetzes (Forschungsförderung) über die Exzellenzinitiative des Bundes und der Länder zur Förderung von Wissenschaft und Forschung an deutschen Hochschulen - Exzellenzvereinbarung (ExV) (http://www.wissenschaftsrat.de/ download/Exzellenziniative_Dokumente/BLK-ExIni.pdf, Zugriff: 20.01.2014)

Bundesministerium für Bildung und Forschung (2012). Die Exzellenzinitiative ist ein Schub für unsere Wissenschaft. In: Pressemitteilung 075/2012 (http://www.bmbf.de/ press/3296.php, Zugriff: 9.06.2013)

Carnevale, Anthony. P. \& Rose, Stephen J. (2003). Socioeconomic Status, Race/Ethnicity, and Selective College Admissions. In: Report for the Century Foundation. New York

Datcher-Loury, Linda \& Garman, David (1995). College Selectivity and Earnings. In: Journal of Labor Economics, H.13, S.289-308 
Denzler, Stefan \& Wolter, Stefan C. (2010). Der Einfluss des lokalen Hochschulangebots auf die Studienwahl. In: Zeitschrift für Erziehungswissenschaft, H.13, S.683-706

Die Welt (2012). Millionen für Elite-Universitäten in München. (http://www.welt.de/regionales/muenchen/article106620714/Millionen-fuer-EliteUniversitaeten -in-Muenchen.html, Zugriff: 22.01.2015)

Diefenbach, Heike (2009): Die Theorie der Rationalen Wahl oder „Rational Choice“-Theorie (RCT). In Ditmar Brock, Matthias Junge \& Heike Diefenbach u. a (Hg.): Soziologische Paradigmen nach Talcott Parsons 2009, pp 239-290

Diekmann, Andreas \& Voss Thomas. Die Theorie rationalen Handelns. Stand und Perspektiven. In: Andreas Diekmann \& Thomas Voss (Hg.), Rational-Choice-Theorie in den Sozialwissenschaften. Anwendungen und Probleme. München: Oldenbourg, S. 13-29.

Eff, E. Anthon \& Klein, Christopher C., Kyle, Reuben (2012). Identifying the Best Buys in U.S. Higher Education. In: Research in Higher Education; H.53, S.860-887

Erikson, Robert \& Jonsson, Jan. O. (1996). Introduction: Explaining Class Inequality in Education: The Swedish Test Case. In: Robert Erikson \& Jan O. Jonsson (Hg.), Can Education Be Equalized? The Swedish Case in Comparative Perspective. Boulder: Westview Press, S.1-63

Esping-Andersen, Gøsta (2004). Untying the Gordian Knot of Social Inheritance. In: Research in Social Stratification, H.21, S.115-138

Esser, Hartmut (1999). Soziologie. Spezielle Grundlagen. Situationslogik und Handeln, Bd. 1. Frankfurt am Main: Campus Verlag

Fleming, Jaqueline (1990). Standardized Test Scores and the Black College Environment. In: Kofi Lomotey (Hg.), Going to School: The African-American Experience. Albany: SUNY Press, S.143-152

Ganzeboom, Harry. B. G, \& Graaf, Paul de. Treiman, Donald J. (1992). A Standard International Socio-Economic Index of Occupational Status. In: Social Science Research, H.21, S.1-56

Halaby, Charles N. (2004). Panel Models in Sociological Research: Theory into Practice. In: Annual Review of Sociology, H.30, S.507-544

Hartmann, Michael (2012). Funktionale oder vertikale Differenzierung - Die Folgen der Exzellenzinitiative. In: Ulf Banscherus, Klemens Himpele \& Andreas Keller (Hg.): Gewerkschaft Erziehung und Wissenschaft: Gut - besser- exzellent? Qualität von Forschung, Lehre und Studium entwickeln. Bielefeld: Bertelsmann, S.29-41

Hartog, Joop \& Sun, Yuze, Ding, Xiaohao (2010). University Rank and Bachelor's Labour Market Positions in China. In: Economics of Education Review 19, 971-979.

Hazelkorn, Ellen (2007). Impact and Influence of League Tables and Ranking Systems on Higher Education Decision-Making. In: Higher Education Management and Policy, H.19, S.1-2

Helbig, Marcel \& Ulbricht, Lena (2010). Perfekte Passung - Finden die besten Hochschulen die besten Studenten? In: Sabine Trepte \& Marcus Verbeet $(\mathrm{Hg}$.$) , Allgemeinbildung in$ Deutschland. Wiesbaden: VS-Verlag, S.107-118

Horstschräer, Julia (2012). University Rankings in Action? The Importance of Rankings and an Excellence Competition for University Choice of High-Ability Students. In: Economics of Education Review, H.31, S.1162-1176

Karabel, Jerome \& Astin, Alexander W. (1975). Social Class, Academic Ability, and College "Quality". In: Social Forces, H.53, S.381-398 
Leibfried, Stephan \& Stock, Günther (2010). Die Exzellenzgalerie aus der Vogelperspektive: 2005-2009. In: Stephan Leibfried (Hg.), Die Exzellenzinitiative. Zwischenbilanz und Perspektiven, H. 15. Frankfurt: Campus Verlag

Lörz, Markus \& Quast, Heiko (2011). Soziale Ungleichheit bei der Hochschulwahl. In: HIS Magazin, H.4, S.1-4

Marginson, Simon (2009). The Knowledge Economy and Higher Education: Rankings and Classifications, Research Metrics and Learning Outcomes Measures as a System for Regulating the Value of Knowledge. In: Higher Education Management and Policy, H.21, S.1-15

McDonough, Patricia M. \& Lising Antonio, Anthony, Walpole, Mary Beth, Xóchitl Pérez, Leonor (1998). College Rankings: Democratized College Knowledge for Whom? In: Research in Higher Education, H.39, S.513-537

McPherson, Michael S. \& Schapiro, Morton Owen (1991). Keeping College Affordable: Government and Educational Opportunity. Washington: The Brookings Institution

Menon, Maria Eliophotou \& Saiti, Anna. Socratous, Michalis (2007). Rationality, Information Search and Choice in Higher Education: Evidence from Greece. In: Higher Education, H.54, S.705-721

Meredith, Marc (2004). Why Do Universities Compete in the Ratings Game? An Empirical Analysis of the Effects of the U.S. News and World Report College Rankings. In: Research in Higher Education, H.45, S.443-461

Monks, James \& Ehrenberg, Ronald G. (1999). The Impact of U.S. News \& World Report College Rankings on Admission Outcomes and Pricing Policies at Selective Private Institutions. In: Cornell Higher Education Research Institute (CHERI), Paper 1

Morgan, Stephen. L. \& Winship, Christopher Winship (2010). Counterfactuals and Causal Inferenz. Methods and Prinziples for Social Research. New York: Cambridge University Press

Müller, Walter \& Pollak, Reinhard, Reimer, David, Schindler, Steffen (2009). Hochschulbildung und soziale Ungleichheit. In: Rolf Becker \& Andreas Hadjar (Hg.), Lehrbuch der Bildungssoziologie. Wiesbaden: VS Verlag, S.281-320

Opp, Karl-Dieter (2010). Kausalität als Gegenstand der Sozialwissenschaften und der multivariaten Statistik. In: Christof Wolf \& Henning Best (Hg.), Handbuch der Sozialwissenschaftlichen Datenanalyse. Wiesbaden: VS Verlag, S.9-39

Sarrico, Cláudia S. \& Hogan, Suzanne M., Dyson, Robert. G., Athanassopoulos, Antreas. D. (1997). Data Envelopment Analysis and University Selection. In: Journal of the Operational Research Society, H.48, S1163-1177

Schindler, Steffen \& Lörz, Markus (2012). Mechanisms of Social Inequality Development: Primary and Secondary Effects in the Transition to Tertiary Education between 1976 and 2005. In: European Sociological Review, H.28, S.647-660

Schindler, Steffen \& Reimer, David (2010). Primäre und sekundäre Effekte der sozialen Herkunft beim Übergang in die Hochschulbildung. In: Kölner Zeitschrift für Soziologie und Sozialpsychologie, H.62, S.623-653.

Schindler, Steffen (2014): Wege zur Studienberechtigung - Wege ins Studium? Eine Analyse sozialer Inklusions-und Ablenkungsprozesse. Wiesbaden: Springer VS.

Sojkin, Bogdan \& Bartkowiak, Paweł, Skuza, Agnieszka (2012). Determinants of Higher Education Choices and Student Satisfaction: The Case of Poland. In: Higher Education, H.63, S.565-581 
Spence, Michael (1973). Job Market Signaling. In: Quarterly Journal of Economics, H.87, S.355-374

Tavares, Orlando \& Cardoso, Sónia ( 2013). Enrolment Choices in Portuguese Higher

Education: Do Students Behave as Rational Consumers? In: Higher Education, H.66, S.297309

Valencia, Richard R.\& Aburto, Sofia (1991). The Uses and Abuses of Educational Testing: Chicanos as a Case in Point. In: Richard Valencia (Hg.), Chicano School Failure and Success. Research and Policy Agendas for the 1990s. Philadelphia: Falmer Press, S.203-251

Williams, Gareth \& Filippakou, Ourania (2010). Higher Education and UK Elite Formation in the Twentieth Century. In: Higher Education, H.59, S.1-20

Willich, Julia \& Buck, Daniel, Heine, Christoph, Sommer, Dieter (2011). Studienanfänger im Wintersemester 2009/10. Wege zum Studium, Studien- und Hochschulwahl, Situation bei Studienbeginn. In: HIS: Forum Hochschule, H.F06/2011. Hannover: HIS. 